\title{
Surface theorem for the Chern-Simons axion coupling
}

\author{
Olsen, Thomas; Taherinejad, Maryam ; Vanderbilt, David ; Souza, Ivo
}

Published in:

Physical Review B

Link to article, DOI:

10.1103/PhysRevB.95.075137

Publication date:

2017

Document Version

Publisher's PDF, also known as Version of record

Link back to DTU Orbit

Citation (APA):

Olsen, T., Taherinejad, M., Vanderbilt, D., \& Souza, I. (2017). Surface theorem for the Chern-Simons axion coupling. Physical Review B, 95, [075137]. https://doi.org/10.1103/PhysRevB.95.075137

\section{General rights}

Copyright and moral rights for the publications made accessible in the public portal are retained by the authors and/or other copyright owners and it is a condition of accessing publications that users recognise and abide by the legal requirements associated with these rights.

- Users may download and print one copy of any publication from the public portal for the purpose of private study or research.

- You may not further distribute the material or use it for any profit-making activity or commercial gain

- You may freely distribute the URL identifying the publication in the public portal

If you believe that this document breaches copyright please contact us providing details, and we will remove access to the work immediately and investigate your claim. 


\title{
Surface theorem for the Chern-Simons axion coupling
}

\author{
Thomas Olsen, ${ }^{1,2, *}$ Maryam Taherinejad, ${ }^{3}$ David Vanderbilt, ${ }^{4}$ and Ivo Souza ${ }^{5,6}$ \\ ${ }^{1}$ Center for Atomic-Scale Materials Design, Department of Physics, Technical University of Denmark, 2820 Kongens Lyngby, Denmark \\ ${ }^{2}$ Center for Nanostructured Graphene (CNG), Department of Nanotechnology, Technical University of Denmark, \\ 2820 Kongens Lyngby, Denmark \\ ${ }^{3}$ Materials Theory, ETH Zürich, Wolfgang-Pauli-Strasse 27, CH-8093 Zürich, Switzerland \\ ${ }^{4}$ Department of Physics and Astronomy, Rutgers University, Piscataway, New Jersey 08854-8019, USA \\ ${ }^{5}$ Centro de Física de Materiales, Universidad del País Vasco, 20018 San Sebastián, Spain \\ ${ }^{6}$ Ikerbasque Foundation, 48013 Bilbao, Spain
}

(Received 25 November 2016; published 21 February 2017)

\begin{abstract}
The Chern-Simons axion coupling of a bulk insulator is only defined modulo a quantum of $e^{2} / h$. The quantized part of the coupling is uniquely defined for a bounded insulating sample, but it depends on the specific surface termination. Working in a slab geometry and representing the valence bands in terms of hybrid Wannier functions, we show how to determine that quantized part from the excess Chern number of the hybrid Wannier sheets located near the surface of the slab. The procedure is illustrated for a tight-binding model consisting of coupled quantum anomalous Hall layers. By slowly modulating the model parameters it is possible to transfer one unit of Chern number from the bottom to the top surface over the course of a cyclic evolution of the bulk Hamiltonian, changing the surface anomalous Hall conductivity by a quantum of conductance $e^{2} / h$. When the evolution of the surface Hamiltonian is also cyclic, the Chern pumping is obstructed by chiral touchings between valence and conduction surface bands.
\end{abstract}

DOI: 10.1103/PhysRevB.95.075137

\section{INTRODUCTION}

The axion field was originally introduced as a strategy for resolving the nonviolation of time reversal $(\mathcal{T})$ and spatial inversion $(\mathcal{P})$ symmetry in quantum chromodynamics (QCD) [1]. Specifically, the gauge symmetry of QCD allows a term in the Lagrangian that is a non-Abelian version of

$$
\mathcal{L}_{\theta}=\frac{e^{2}}{h} \frac{\theta}{2 \pi} \boldsymbol{E} \cdot \boldsymbol{B},
$$

where $\theta$ is a fixed dimensionless parameter, and $\boldsymbol{E}$ and $\boldsymbol{B}$ are the electric and magnetic fields. Since $\boldsymbol{B}$ and $\boldsymbol{E}$ are odd under $\mathcal{T}$ and $\mathcal{P}$, respectively, these symmetries are individually broken by $\mathcal{L}_{\theta}$. The most striking consequence of such a term is the prediction of a finite electric dipole moment for the neutron, but recent experimental bounds on this quantity [2] restrict $|\theta|$ to be less than about $10^{-9}$. Such fine tuning is regarded as unnatural, and in order to circumvent it Peccei and Quinn showed that promoting $\theta$ to a dynamical field leads to a vanishing vacuum expectation value for the field $(\theta=$ $\langle\hat{\theta}\rangle=0$ ), elegantly restoring $\mathcal{T}$ and $\mathcal{P}$ symmetry in QCD [1]. Excitations of the field naturally give rise to a new massive particle known as the axion $[3,4]$. The axion has never been observed, but is presently regarded as an important candidate for dark matter [5].

The classical field theories resulting from the inclusion of the $\mathcal{L}_{\theta}$ term have several interesting properties and have been investigated in a number of different contexts [6]. A most remarkable property is that the theory is invariant under $\theta \rightarrow$ $\theta+2 \pi$, so that $\theta$ is best viewed as a phase. This is related to the fact that $\boldsymbol{E} \cdot \boldsymbol{B}=\varepsilon_{a b c d} F^{a b} F^{c d} / 8$ is proportional to the second Chern class of the gauge field. Hence, the integral of $\mathcal{L}_{\theta} / \theta$

\footnotetext{
*tolsen@fysik.dtu.dk
}

over a closed manifold yields an integer that represents the winding number of the field configuration [7]. In addition, the evaluation of any correlation function involves a path integral of $\exp \left[i \int d x^{4} \mathcal{L}_{\theta}(x)\right]$, which becomes $e^{i n \theta}=e^{i n(\theta+2 \pi)}$ for any field configuration that vanishes at infinity.

Another interesting interpretation of $\theta$ relates to the nontrivial vacuum structure allowed by non-Abelian gauge theories. Different vacua are then classified by the winding number $n$ of classical field configurations, and the vacuum state is taken as $|\theta\rangle=\sum_{n} e^{i \theta n}|n\rangle$ [8]. In this context $\theta$ is referred to as the vacuum angle, and can be regarded as the "Bloch momentum" associated with a particular state on the "lattice" of topologically distinct field configurations.

In a condensed-matter setting, the analog of a vacuum state is the electronic ground state of an insulator. In that case $\theta$ is a material property determined by the electronic structure. If we allow the presence of $\mathcal{L}_{\theta}$ in the Lagrangian, Maxwell's equations acquire additional $\theta$-dependent terms. Since $\mathcal{L}_{\theta}$ with constant $\theta$ can be rewritten as a total derivative, only regions of changing $\theta$ can give rise to physically observable effects, as long as the gauge fields are treated classically. If one now considers an insulating boundary separating two materials characterized by different $\theta$ values, it is straightforward to show that the interface will support an in-plane current given by $[9,10]$

$$
j_{i}=\widetilde{j}_{i}+\frac{e^{2}}{h} \frac{\Delta \theta}{2 \pi} \varepsilon_{i j} E^{j} .
$$

The first term is the usual current appearing in Maxwell's equations, and the second is an additional "axial" current that arises due to the change $\Delta \theta$ across the boundary. We now see that changing $\Delta \theta \rightarrow \Delta \theta+2 \pi$ amounts to adding a quantum of anomalous Hall conductivity (AHC) to the interface. Conversely, if the AHC at an insulating interface is known, it becomes possible to assign a definite value to $\Delta \theta$. 
The transformation properties of $\boldsymbol{E}$ and $\boldsymbol{B}$ in Eq. (1) indicate that $\theta$ is odd under $\mathcal{T}$ and $\mathcal{P}$. Due to the $2 \pi$ ambiguity, those symmetries allow for $\theta=\pi$ as well as $\theta=0$, leading to a $\mathbb{Z}_{2}$ topological classification. It is well known that when $\mathcal{T}$ symmetry is present the value $\theta=\pi$ describes strong topological insulators, while topologically trivial insulators have $\theta=0$ [10]. In the case in which $\mathcal{P}$ is conserved but not $\mathcal{T}$, the term "axion insulator" has sometimes been used $[11,12]$ to describe the topological phase with $\theta=\pi$. More generally, any magnetic point group that contains a proper rotation composed with $\mathcal{T}$, or an improper rotation not composed with $\mathcal{T}$, supports a $\mathbb{Z}_{2}$ classification with $\theta$ constrained to be 0 or $\pi[13]$.

The existence or nature of topologically protected boundary states depends on the symmetry protecting $\theta=\pi$. For example, when $\mathcal{T}$ remains unbroken at the surface of a strong topological insulator, that surface is guaranteed to harbor an odd number of metallic Dirac cones, with a half-quantized surface AHC that exactly cancels the axial current [14]. In contrast, the surface of an axion insulator automatically breaks $\mathcal{P}$, so that surface states are not protected. In the case of mirror symmetry, metallic states are only protected on mirror-preserving surfaces, while surfaces that break mirror symmetry can be insulating and display a half-quantized AHC.

The term $\mathcal{L}_{\theta}$ given by Eq. (1) is not present a priori in the action of condensed-matter systems, where it should be regarded as an effective term that sometimes provides a useful description. Indeed, such a term appears when the electrons are integrated out of a generic solid state partition function in four dimensions, followed by dimensional reduction [10]. That procedure leads to the expression

$$
\theta_{\mathrm{CS}}=-\frac{1}{4 \pi} \int d \boldsymbol{k} \epsilon_{i j l} \operatorname{Tr}\left[A_{\boldsymbol{k}}^{i} \partial_{k_{j}} A_{\boldsymbol{k}}^{l}-i \frac{2}{3} A_{\boldsymbol{k}}^{i} A_{\boldsymbol{k}}^{j} A_{\boldsymbol{k}}^{l}\right]
$$

for the axion coupling strength. Here

$$
A_{k n m}^{i}=i\left\langle u_{k n} \mid \partial_{k_{i}} u_{k m}\right\rangle
$$

is the Berry connection matrix in Cartesian direction $i$, where $\left|u_{k n}\right\rangle$ is the cell-periodic part of the Bloch function $\left|\psi_{k n}\right\rangle$ of the $n$th occupied band, $\langle\ldots\rangle$ denotes an integration over one crystal cell, and the trace is over occupied bands. The integral in $d \boldsymbol{k}$ is over the Brillouin zone (BZ), and the integrand is known as the Chern-Simons (CS) 3-form.

The form of Eq. (1) suggests a close relation between $\theta$ and the linear magnetoelectric response tensor defined as $\alpha_{i j}=\left(\partial P_{i} / \partial B_{j}\right)_{E}=\left(\partial M_{j} / \partial E_{i}\right)_{\boldsymbol{B}}$, where $\boldsymbol{P}$ and $\boldsymbol{M}$ are the macroscopic polarization and magnetization, respectively. With $\widetilde{\alpha}$ the traceless part of $\alpha$, the relation reads

$$
\begin{aligned}
\alpha_{i j} & =\widetilde{\alpha}_{i j}+\bar{\alpha} \delta_{i j}, \\
\bar{\alpha} & =\frac{e^{2}}{h} \frac{\theta}{2 \pi} .
\end{aligned}
$$

This defines $\theta$ in terms of the trace piece $\bar{\alpha}$, also known as the "axion magnetoelectric coupling".

The magnetoelectric response of a solid can be decomposed into spin and orbital contributions on one hand, and frozen-ion and lattice-mediated contributions on the other. The frozen-ion orbital part of the response was calculated for generic band insulators in Refs. $[15,16]$. It was found that the trace piece takes the form $\bar{\alpha}_{\text {orb }}=\left(e^{2} / 2 \pi h\right)\left(\theta_{\mathrm{CS}}+\theta_{\mathrm{cg}}\right)$ where, in addition to $\theta_{\mathrm{CS}}$ given by Eq. (3), there is a cross-gap term $\theta_{\mathrm{cg}}$ that couples occupied and empty bands. Among all of the above terms, $\theta_{\mathrm{CS}}$ is the only term with a $2 \pi$ ambiguity. It is thus sufficient, for the purpose of establishing the surface theorem, to focus on the Chern-Simons axion (CSA) coupling

$$
\bar{\alpha}_{\mathrm{CS}}=\frac{e^{2}}{h} \frac{\theta_{\mathrm{CS}}}{2 \pi} .
$$

Typical ground state properties of a band insulator (e.g., the charge density and total energy) are invariant under any unitary "gauge transformation"

$$
\left|u_{k n}\right\rangle \rightarrow \sum_{m}\left|u_{k m}\right\rangle U_{k m n}
$$

among the occupied Bloch orbitals. The $2 \pi$ ambiguity in $\theta_{\mathrm{CS}}$ comes about because Eq. (3) changes by integer multiples of $2 \pi$ under certain gauge transformations. The CSA coupling will then change by an integer multiple of the quantum of conductance. This is another manifestation of Eq. (2): changing $\theta_{\mathrm{CS}}$ by a multiple of $2 \pi$ amounts to adding quantum anomalous Hall layers at the surface, without modifying the bulk [17].

Another property of band insulators that behaves in this way is the bulk polarization $\boldsymbol{P}$, whose electronic part can be expressed as a Berry phase [18]. The Berry phase may also change by an integer multiple of $2 \pi$ under unitary transformations, and the quantum of ambiguity in the bulk definition of $\boldsymbol{P} \cdot \hat{\boldsymbol{n}}$ can be resolved by taking into account quantized contributions of $e / A_{\text {cell }}$ to the surface charge density associated with an insulating surface with orientation $\hat{\boldsymbol{n}}$ [19].

In fact, $\bar{\alpha}_{\mathrm{CS}}$ and $\boldsymbol{P}$ are related in more than one respect. First of all, $\bar{\alpha}_{\mathrm{CS}}$ is in a sense the natural three-dimensional generalization of the polarization in one-dimensional systems, and is sometimes referred to as the "magnetoelectric polarization" [10]. For any closed manifold of odd dimension $d$, there exists a Chern-Simons $d$-form, which is a functional of the $U(N)$ connection $A_{k m n}^{i}$. The 1-form is simply the trace of the connection, and its BZ integral (the Berry phase) is proportional to the polarization. Similarly, the BZ integral of the Chern-Simons 3-form yields $\theta_{\mathrm{CS}}$, which is proportional to the CSA coupling. Second, the $e^{2} / h$ ambiguity in $\bar{\alpha}_{\mathrm{CS}}$ follows from the $e / A_{\text {cell }}$ ambiguity in $\boldsymbol{P} \cdot \hat{\boldsymbol{n}}$ [16]. This is perhaps surprising, since changes in polarization are well defined whenever different states of polarization can be connected by an adiabatic path. However, as pointed out in Ref. [16], if one tries to define the CSA coupling as a response of $P_{i}$ to a smoothly increasing $B_{i}$, a problem arises in that the smallest value of $\Delta B_{i}$ compatible with the lattice periodicity corresponds to a quantum of magnetic flux through the unit cell. The best one can do is to define $\bar{\alpha}_{\mathrm{CS}}=\Delta P_{i} / \Delta B_{i}$ for a single flux quantum, i.e., $\Delta B_{i}=h / e A_{\text {cell. }}$ Then the gauge ambiguity of $e / A_{\text {cell }}$ in $P_{i}$ implies a gauge ambiguity of $e^{2} / h$ in $\bar{\alpha}_{\mathrm{CS}}$.

The surface theorem for polarization was derived in Ref. [19]. It states that the macroscopic charge per surface unit cell at an insulating surface of a crystalline insulator has, in units of $e$, a noninteger part that only depends on the bulk polarization, and an integer part that is fixed by the surface termination. 
In this work, we demonstrate a similar surface theorem for the CSA coupling of Eq. (6). We take a bulk insulator with a given $\theta_{\mathrm{CS}}(\bmod 2 \pi)$, consider a specific insulating surface termination in a slab geometry, and show how to use the knowledge about the surface Hamiltonian to determine the CSA coupling exactly, not just up to a quantum of $e^{2} / h$.

An important step towards that result was taken in Ref. [20], where Eq. (3) was recast in terms of hybrid Wannier functions (HWFs), and then used to study a "quantum CSA pump": a cyclic evolution of a bulk crystal in which $\theta_{\mathrm{CS}}$ evolves continuously from some initial value $\theta_{\mathrm{CS}}^{i}$ to reach $\theta_{\mathrm{CS}}^{i}+2 \pi$ at the end of the cycle. In the HWF representation, the pumping process is transparent: the "Wannier sheets" carry quantized amounts of Berry-curvature flux, and a $2 \pi$ quantum gets transferred across each unit cell via a sequence of sheet-touching events. These ideas play a central role in the present work, where the HWF representation will be used extensively.

The paper is organized as follows. In Sec. II A we establish the basic definitions and conventions used in this work, and in Sec. II B we review the expression of Ref. [20] for $\theta_{\mathrm{CS}}$ in terms of the bulk HWFs. The goal of the ensuing subsections is to evaluate the CSA coupling of a thick slab in the HWF basis. The end result is the surface theorem of Eq. (44), whereby the noninteger part of $\theta_{\text {slab }} / 2 \pi$ is given by the bulk expression in terms of the HWFs in a unit cell deep inside the slab, and the integer part by the net Chern number of the excess Wannier sheets near the surface. In order to establish this result, we start in Sec. II C from an expression for the CSA coupling of a finite crystallite; then in Sec. IID we derive from it the corresponding expression for a slab; finally in Sec. IIE we consider its limiting form for a thick slab. At every step all surface contributions are carefully accounted for, leading to Eq. (44) for insulating slabs with vanishing Chern number. To validate the derivation and illustrate the surface theorem, in Sec. III we study numerically a layered tight-binding model that realizes a quantum CSA pump. We find that in a semi-infinite geometry, when the surface also undergoes a cyclic evolution, the pumping is obstructed by the appearance of "surface Weyl points" that transfer quanta of Berry-curvature flux between the valence and conduction bands. Some additional features of the model are discussed in two appendices. Appendix A deals with the quantization of the CSA coupling by mirror symmetry at isolated points along the pumping cycle, and in Appendix B we map the second Chern number over an augmented parameter space.

\section{CSA COUPLING IN THE HYBRID WANNIER REPRESENTATION}

\section{A. Definitions and conventions}

The CSA coupling strength $\theta_{\mathrm{CS}}$ of an infinite $3 \mathrm{D}$ crystal was defined in Eq. (3). In the remainder of the paper we will change notation and denote it as $\theta_{3}$ instead. The reason is that in order to establish the precise relation between the bulk CSA coupling and the observable magnetoelectric response of a bounded macroscopic sample, we also want to consider systems of reduced dimensionality $d$, namely slabs $(d=2)$ and crystallites $(d=0)$, and define for them corresponding quantities $\theta_{2}$ and $\theta_{0}$. In each case $\theta_{d}$ is defined as the extensive CSA coupling with units of volume, divided by the cell "volume" along the periodic directions, so that $\theta_{d}$ has units of $[L]^{3-d}: \theta_{0}$ grows with the volume of the crystallite, $\theta_{2}$ with the slab thickness, and $\theta_{3} \equiv \theta_{\mathrm{CS}}$ is an intensive bulk quantity.

Let us begin with the bulk crystal, and take $\hat{z}$ along a primitive reciprocal lattice vector $\boldsymbol{b}_{3}$. The primitive cell subtended by $\boldsymbol{a}_{1}, \boldsymbol{a}_{2}$, and $\boldsymbol{a}_{3}$ is a prism with the base $\left(\boldsymbol{a}_{1}, \boldsymbol{a}_{2}\right)$ lying on the $(x, y)$ plane and height

$$
c=\frac{2 \pi}{\left|\boldsymbol{b}_{3}\right|} .
$$

The Bloch functions are normalized to one primitive cell, $\int_{V_{\text {cell }}} d \boldsymbol{r}\left|\psi_{\boldsymbol{k} n}(\boldsymbol{r})\right|^{2}=1$ with $V_{\text {cell }}=\left(\boldsymbol{a}_{1} \times \boldsymbol{a}_{2}\right) \cdot \boldsymbol{a}_{3}$.

In order to define HWFs for lattices of arbitrary symmetry, it is convenient to work in reduced coordinates. For example, the wave vector $\boldsymbol{k}$ is expanded as

$$
\boldsymbol{k}=k_{1} \boldsymbol{b}_{1}+k_{2} \boldsymbol{b}_{2}+k_{3} \boldsymbol{b}_{3} .
$$

In the HWF representation, the $\hat{z}$ direction is treated differently from $\hat{\boldsymbol{x}}$ and $\hat{\boldsymbol{y}}$. Defining $\langle f \mid g\rangle$ as the integral over all $z$ and over one cell on the basal plane, the orthonormality relation for the Bloch states reads

$$
\left\langle\psi_{\left(k_{1}, k_{2}, k_{3}\right) n} \mid \psi_{\left(k_{1}^{\prime}, k_{2}^{\prime}, k_{3}^{\prime}\right) m}\right\rangle=N_{3} \delta_{k_{1} k_{1}^{\prime}} \delta_{k_{2} k_{2}^{\prime}} \delta_{k_{3} k_{3}^{\prime}} \delta_{n m},
$$

where for the moment we work on a discrete uniform $\boldsymbol{k}$ mesh, with $N_{3}$ distinct values of $k_{3}$ in the BZ. The bulk HWFs are defined as

$$
\left|\mathfrak{h}_{\left(k_{1}, k_{2}\right) l n}\right\rangle=\frac{1}{N_{3}} \sum_{k_{3}} e^{-i 2 \pi k_{3} l}\left|\psi_{\left(k_{1}, k_{2}, k_{3}\right) n}\right\rangle,
$$

where $l$ labels the cells along $\boldsymbol{a}_{3}$. The HWFs are localized (Wannier-like) along $\boldsymbol{b}_{3} \| \hat{\boldsymbol{z}}$, but remain extended (Bloch-like) along $\hat{\boldsymbol{x}}$ and $\hat{\boldsymbol{y}}$. Using Eq. (10) we obtain $\left\langle\mathfrak{h}_{\left(k_{1}, k_{2}\right) l n} \mid \mathfrak{h}_{\left(k_{1}^{\prime}, k_{2}^{\prime}\right) l^{\prime} m}\right\rangle=$ $\delta_{k_{1} k_{1}^{\prime}} \delta_{k_{2} k_{2}^{\prime}} \delta_{l l^{\prime}} \delta_{n m}$.

In Sec. II D we will define HWFs for slabs with the surface normal along $\boldsymbol{b}_{3}$. The wave vector labels of such HWFs are most naturally chosen in the $2 \mathrm{D} \mathrm{BZ}$ of an unreconstructed surface. This "projected BZ" is spanned by the vectors

$$
\begin{aligned}
& \boldsymbol{B}_{1}=2 \pi \frac{\boldsymbol{a}_{2} \times \hat{z}}{\left(\boldsymbol{a}_{1} \times \boldsymbol{a}_{2}\right) \cdot \hat{z}}, \\
& \boldsymbol{B}_{2}=2 \pi \frac{\hat{z} \times \boldsymbol{a}_{1}}{\left(\boldsymbol{a}_{1} \times \boldsymbol{a}_{2}\right) \cdot \hat{z}},
\end{aligned}
$$

which are the duals of $\boldsymbol{a}_{1}$ and $\boldsymbol{a}_{2}$ on the $(x, y)$ plane: $\boldsymbol{B}_{i} \cdot \boldsymbol{a}_{j}=$ $2 \pi \delta_{i j}$ for $i, j=1,2$. A vector $\boldsymbol{k}$ in the BZ of the bulk crystal can be expressed as $\boldsymbol{k}=\boldsymbol{\kappa}+k_{z} \hat{\boldsymbol{z}}$, where

$$
\boldsymbol{\kappa}=k_{x} \hat{\boldsymbol{x}}+k_{y} \hat{\boldsymbol{y}}=k_{1} \boldsymbol{B}_{1}+k_{2} \boldsymbol{B}_{2}
$$

belongs to the projected $\mathrm{BZ}$, and $k_{1}$ and $k_{2}$ are the same as in Eq. (9). Thus, both the slab HWFs and the bulk HWFs can be labeled by wave vectors in the projected BZ. With this notation, which will be useful for establishing the surface theorem in Sec. II E, Eq. (11) becomes

$$
\begin{aligned}
\left|\mathfrak{h}_{\kappa l n}\right\rangle & =\int_{0}^{1} d k_{3} e^{-i 2 \pi k_{3} l}\left|\psi_{\left(\kappa, k_{3}\right) n}\right\rangle, \\
\left|h_{\kappa l n}\right\rangle & =e^{-i \kappa \cdot \boldsymbol{r}}\left|\mathfrak{h}_{\kappa l n}\right\rangle,
\end{aligned}
$$


where we took $N_{3} \rightarrow \infty$ and introduced the cell-periodic part $\left|h_{\kappa l n}\right\rangle$ of a HWF, with in-plane lattice periodicity.

We are interested in constructing HWFs that span the group of $M$ valence bands. The $U(M)$ gauge freedom of Eq. (7) in defining the Bloch states can be employed to make the HWFs maximally localized along $\hat{z}$, in the sense of Ref. [21]. The charge centers of these maximally localized HWFs

$$
z_{\kappa l n}=\left\langle\mathfrak{h}_{\kappa l n}|z| \mathfrak{h}_{\kappa l n}\right\rangle=z_{\kappa 0 n}+l c
$$

form 2D sheets over the projected BZ [22]. Dropping subscripts $\kappa$ for brevity, Berry connection and Berry curvature matrices can be defined over these sheets as

$$
\begin{aligned}
& A_{l n, l^{\prime} m}^{i}=i\left\langle h_{l n} \mid \partial_{k_{i}} h_{l^{\prime} m}\right\rangle=A_{0 n,\left(l^{\prime}-l\right) m}^{i}, \\
& \Omega_{l n, l^{\prime} m}^{i j}=\partial_{k_{i}} A_{l n, l^{\prime} m}^{j}-\partial_{k_{j}} A_{l n, l^{\prime} m}^{i}=\Omega_{0 n,\left(l^{\prime}-l\right) m}^{i j},
\end{aligned}
$$

where $i, j=x, y$.

Assuming that the Wannier sheets do not touch (i.e., there are no degeneracies in the $z_{l n}$ anywhere in the 2D BZ), each sheet is a closed 2D manifold, so that the integral of its Berry curvature (the Berry flux through the 2D BZ) is quantized to $2 \pi$ times an integer by the Chern theorem. These Chern numbers

$$
C_{l n}=\frac{1}{2 \pi} \int d \boldsymbol{\kappa} \Omega_{l n, l n}^{x y}
$$

are clearly independent of the layer index, $C_{l n}=C_{0 n}$.

We shall encounter situations in Sec. III where pairs of Wannier sheets touch at isolated points in the $2 \mathrm{D} \mathrm{BZ}$ as a parameter $\phi$ in the Hamiltonian is varied. When that happens, the Chern numbers of the two sheets change by equal and opposite amounts $\pm \chi$, where $\chi$ is the chiral charge of the degeneracy point ("Weyl point") in the 3D parameter space $\left(k_{x}, k_{y}, \phi\right)$.

\section{B. CSA coupling $\theta_{3}$ of a bulk crystal}

We begin by summarizing the results of Ref. [20], where the bulk CSA coupling was expressed in terms of maximally localized HWFs as [23]

$$
\begin{aligned}
\theta_{3} & =\theta_{z \Omega}+\theta_{\Delta x y}, \\
\theta_{z \Omega} & =-\frac{1}{c} \int d \kappa \sum_{n} z_{0 n} \Omega_{0 n, 0 n}^{x y}, \\
\theta_{\Delta x y} & =-\frac{i}{c} \int d \kappa \sum_{l n m}\left(z_{l m}-z_{0 n}\right) A_{0 n, l m}^{x} A_{l m, 0 n}^{y},
\end{aligned}
$$

where the integral is over the $2 \mathrm{D}$ projected BZ. (Henceforth summations over indices $l, m$, and $n$ run over all occupied orbitals, unless stated otherwise.) If the lattice has monoclinic symmetry or higher and $\boldsymbol{a}_{3}$ is the unique axis, then $c=\left|\boldsymbol{a}_{3}\right|$. In the general case, $c$ is given by Eq. (8).

The maximally localized HWF gauge is unique, except for (i) a $U(1)$ gauge freedom with respect to $\kappa$ on each sheet, and (ii) the residual freedom to choose which Wannier sheets belong to the home cell $l=0$. Regarding (i), it is straightforward to verify that both terms in Eq. (18) are gauge-invariant in this sense (for $\theta_{\Delta x y}$ this follows because only the diagonal elements of $A_{0 n, l m}^{i}$ are affected). Regarding (ii), if a different choice is made such that entire sheets $z_{l n}$ get shifted by $c$ for some $n, \theta_{z \Omega}$ changes by $-2 \pi C_{0 n}$, while $\theta_{\Delta x y}$ is unaffected. Thus, the $\theta_{z \Omega}$ term is the only one that has a potential $2 \pi$ ambiguity.

In Ref. [20], Eq. (18) was derived starting from Eq. (3). One problem with this approach is that Eq. (3) is written in terms of a smooth and periodic gauge, a requirement that, applied to the maximally localized HWF gauge, is incompatible with the existence of Wannier sheets having nonzero Chern numbers. Thus, strictly speaking Eq. (18) has only been proven to be valid for crystals in which all the $C_{0 n}$ vanish. On the other hand, it was shown in the same work that nonzero $C_{0 n}$ values must occur along any cycle that pumps a quantum of CSA coupling.

In the following, we shall take a different route to arrive at Eq. (18), proving the surface theorem along the way. Instead of working from the outset with a bulk crystal and using Eq. (3), we start from the CSA coupling of a finite crystallite, which is given by Eq. (20) below without any $2 \pi$ ambiguity. By carefully taking the thermodynamic limit, first in two directions (slab geometry) and finally in the third, we will demonstrate that Eq. (18) remains valid even when some of the Wannier sheets have nonzero Chern numbers, provided that the unit-cell sum of those numbers vanishes:

$$
\sum_{n} C_{0 n}=0
$$

This condition, needed to ensure that the nonquantized part of $\theta_{3}$ is independent of the choice of origin for the $z$ axis, is equivalent to the statement that the Chern index along $z$ of the valence-band manifold vanishes [20]. In fact, in the present work we limit ourselves to crystals in which all three Chern indices vanish, in order to avoid potential subtleties associated with bulk quantum anomalous Hall behavior [24].

\section{CSA coupling $\theta_{0}$ of a finite crystallite}

The extensive CSA coupling of a bounded electron system such as a crystallite is given by

$$
\theta_{0}=-8 \pi^{2} \operatorname{Im} \operatorname{Tr}[P x P y P z],
$$

where $P$, the projection operator onto the occupied subspace in the ground state, is expressed in the energy eigenstate representation as $P=\sum_{n}\left|\psi_{n}\right\rangle\left\langle\psi_{n}\right|$.

Equation (20) was obtained in Ref. [15] starting from the basic definition

$$
\boldsymbol{m}(\boldsymbol{E})=-\frac{e}{2} \operatorname{Tr}[P(\boldsymbol{E}) \boldsymbol{r} \times \boldsymbol{v}]
$$

of the orbital magnetic moment of a bounded sample in a finite electric field, with $P(\boldsymbol{E})$ the projection operator onto the field-polarized occupied states. After some manipulations, Eq. (21) was decomposed as [15]

$$
\boldsymbol{m}(\boldsymbol{E})=\widetilde{\boldsymbol{m}}_{\mathrm{LC}}(\boldsymbol{E})+\widetilde{\boldsymbol{m}}_{\mathrm{IC}}(\boldsymbol{E})+\boldsymbol{m}_{\mathrm{CS}}(\boldsymbol{E}) .
$$

At $\boldsymbol{E}=0$, only the first two terms survive (they are known as the "local circulation" and the "itinerant circulation" [25]). At $\boldsymbol{E} \neq 0$ those terms depend on $\boldsymbol{E}$ only through $P(\boldsymbol{E})$, and their contribution to the linear change in $\boldsymbol{m}$ induced by $\boldsymbol{E}$ is in general not parallel to $\boldsymbol{E}$. In contrast, the third term is purely 
isotropic and has an explicit linear dependence on $\boldsymbol{E}$. It takes the form

$$
\boldsymbol{m}_{\mathrm{CS}}(\boldsymbol{E})=\frac{e^{2}}{h} \frac{\theta_{0}(\boldsymbol{E})}{2 \pi} \boldsymbol{E},
$$

with $\theta_{0}(\boldsymbol{E})$ given in terms of $P(\boldsymbol{E})$ by Eq. (20). At linear order in the field, the quantity $\theta_{0}(\boldsymbol{E})$ in Eq. (23) can be replaced with $\theta_{0}(\boldsymbol{E}=0)$, i.e., the ground-state quantity $\theta_{0}$ as originally defined by Eq. (20).

According to the analytical derivations and numerical tests carried out in Ref. [15], in the thermodynamic limit the quantity $\theta_{0} / V$ reduces to the bulk CSA coupling $\theta_{3}$ defined by Eq. (3). However, the fact that $\theta_{3}$ suffers from a $2 \pi$ indeterminacy while $\theta_{0} / V$ does not was not examined further in that work. Because Eq. (20) does not carry a quantum of uncertainty, and is free from the subtleties associated with the use of periodic boundary conditions, we take it as the more fundamental definition of the CSA coupling, from which we will derive expressions for $\theta_{2}$ and $\theta_{3}$ in the HWF representation. The correctness of those expressions will be checked numerically against results obtained directly from Eq. (20).

We begin by constructing for the crystallite a set of occupied orbitals that are maximally localized along $\hat{z}$. This can be done by diagonalizing the operator $P z P[21]$,

$$
P z P\left|\varphi_{n}\right\rangle=z_{n}\left|\varphi_{n}\right\rangle \text {. }
$$

In this representation, Eq. (20) reads

$$
\theta_{0}=-8 \pi^{2} \sum_{n} z_{n} \operatorname{Im}\left\langle\varphi_{n}|x P y| \varphi_{n}\right\rangle
$$

or equivalently

$$
\theta_{0}=8 \pi^{2} \sum_{n} z_{n} \operatorname{Im}\left\langle\varphi_{n}|x Q y| \varphi_{n}\right\rangle
$$

where $Q=1-P$ and we used $\operatorname{Im}\left\langle\varphi_{n}|x y| \varphi_{n}\right\rangle=0$. We shall prefer Eq. (26) for reasons that will become clear in the next subsection.

Before switching to a slab geometry, we note that any welldefined intrinsic property must remain invariant under a rigid translation of the sample, such as

$$
z \rightarrow z+\Delta z
$$

Under this transformation, Eq. (26) changes by

$$
\Delta \theta_{0}=8 \pi^{2} \Delta z \operatorname{Im} \operatorname{Tr}\{P x Q y\} .
$$

It is straightforward to verify that $\Delta \theta_{0}=0$ since $\operatorname{Im} \operatorname{Tr}\{P x P y\}=\operatorname{Tr}\{[P x, P y]\} / 2 i$, and the trace of a commutator over a finite-dimensional Hilbert space vanishes.

\section{CSA coupling $\theta_{2}$ of a slab}

Consider an insulating slab cut from the bulk crystal, with the surface normal oriented along $\boldsymbol{b}_{3} \| \hat{\boldsymbol{z}}$ and thickness $L_{z}=N_{z} c$. We will eventually take the limit $N_{z} \rightarrow \infty$, but at this stage $N_{z}$ can be any positive integer. We wish to find an expression for $\theta_{2}$, the CSA coupling per unit area, starting from Eq. (26). Imagine cutting from the slab a crystallite containing $N \times N$ two-dimensional primitive cells with a net
CSA coupling $\theta_{0}$. By the definition of $\theta_{2}$, we expect that

$$
\frac{\theta_{0}}{N^{2} A_{\text {cell }}} \rightarrow \theta_{2} \text { for } N \rightarrow \infty \text {. }
$$

As we will see, this expectation is fulfilled by our expression for $\theta_{2}$, with the proviso that the net Chern number of the slab vanishes; otherwise $\theta_{2}$ is ill-defined, with its value depending on the choice of origin for the $z$ axis. Such pathological behavior has to do with extensive contributions to $\theta_{0}$ from the sample edges. We shall return to this subtle point at the end of the section, starting with Eq. (38).

To proceed we assume a slab with unreconstructed surfaces, so that the energy eigenstates are Bloch-like along the in-plane directions, with wave vector labels $\boldsymbol{\kappa}$ given by Eq. (13); denoting them by $\left|\psi_{\kappa n}\right\rangle$ and introducing the ground-state projector

$$
P=\sum_{\kappa} P_{\kappa}, \quad P_{\kappa}=\sum_{n}\left|\psi_{\kappa n}\right\rangle\left\langle\psi_{\kappa n}\right|
$$

we can construct a new set of occupied orbitals by diagonalizing $P_{\kappa} z P_{\kappa}$ for each $\kappa$ :

$$
P_{\kappa} z P_{\kappa}\left|\mathfrak{h}_{\kappa n}\right\rangle=z_{\kappa n}\left|\mathfrak{h}_{\kappa n}\right\rangle .
$$

These are the maximally localized HWFs of the slab. For now we assume isolated Wannier sheets $z_{\kappa n}$ with welldefined Chern numbers $C_{n}=(1 / 2 \pi) \int d \kappa \Omega_{\kappa n n}^{x y}$; the role of degeneracies will be considered later.

Comparison with Eq. (24) shows that for $N \rightarrow \infty$ the orbitals $\left|\mathfrak{h}_{\kappa n}\right\rangle$ and $\left|\varphi_{n}\right\rangle$ only differ in the choice of in-plane boundary conditions (periodic versus open). Thus, if edge contributions to $\theta_{0}$ are unimportant we can replace $\left|\varphi_{n}\right\rangle$ with $\left|\mathfrak{h}_{\kappa n}\right\rangle$ in Eq. (26), and using the definition (29) of $\theta_{2}$ we arrive at

$$
\theta_{2}=\int d \kappa \sum_{n} z_{\kappa n} 2 \operatorname{Im}\left\langle\mathfrak{h}_{\kappa n}|x Q y| \mathfrak{h}_{\kappa n}\right\rangle .
$$

The presence of the nonperiodic coordinate operators $x$ and $y$ is not problematic, because they appear in the lattice-periodic combinations $P x Q$ and $Q y P$.

Equation (32) is valid in the maximally localized HWF gauge where $Z_{\kappa n m}=\left\langle\mathfrak{h}_{\kappa n}|z| \mathfrak{h}_{\kappa m}\right\rangle$ is a diagonal matrix: $Z_{\kappa n m}=$ $z_{\kappa n} \delta_{n m}$. In a generic gauge, Eq. (32) must be written in matrix form as

$$
\begin{aligned}
\theta_{2} & =-\int d \boldsymbol{\kappa} \sum_{m n} Z_{\kappa m n} \widetilde{\Omega}_{\kappa n m}^{x y}, \\
\widetilde{\Omega}_{\kappa n m}^{x y} & =-2 \operatorname{Im}\left\langle\mathfrak{h}_{\kappa n}|x Q y| \mathfrak{h}_{\kappa m}\right\rangle .
\end{aligned}
$$

This expression is clearly gauge-invariant, because it is the trace of the product of two gauge-covariant matrices (matrices that change as $M_{\kappa} \rightarrow U_{\kappa}^{\dagger} M_{\kappa} U_{\kappa}$ under a gauge transformation $\left.\left|\mathfrak{h}_{\kappa n}\right\rangle \rightarrow \sum_{m}\left|\mathfrak{h}_{\kappa m}\right\rangle U_{\kappa m n}\right)$.

To proceed we switch momentarily to the Hamiltonian gauge, where the orbitals $\left|\mathfrak{h}_{\kappa n}\right\rangle$ coincide with the energy eigenstates $\left|\psi_{\kappa n}\right\rangle$. Writing $Q=\sum_{\kappa^{\prime}} \sum_{l}^{\text {empty }}\left|\psi_{\kappa^{\prime} l}\right\rangle\left\langle\psi_{\kappa^{\prime} l}\right|$ in Eq. (33b), we see that it only involves off-diagonal matrix elements of the coordinate operators along the periodic directions. Defining the cell-periodic part $\left|u_{\kappa n}\right\rangle=e^{-i \kappa \cdot r}\left|\psi_{\kappa n}\right\rangle$ 
of an eigenstate and using the relation [26]

$$
\left\langle\psi_{\kappa n}|x| \psi_{\kappa^{\prime} m}\right\rangle=i\left\langle u_{\kappa n} \mid \partial_{k_{x}} u_{\kappa m}\right\rangle \delta_{\kappa \kappa^{\prime}}
$$

valid for $n \neq m$ in the Hamiltonian gauge, we find

$$
\begin{aligned}
\widetilde{\Omega}_{\kappa n m}^{x y} & =-2 \operatorname{Im}\left\langle\partial_{k_{x}} u_{\kappa n}\left|Q_{\kappa}\right| \partial_{k_{y}} u_{\kappa m}\right\rangle \\
& =\Omega_{\kappa n m}^{x y}-i\left[A_{\kappa}^{x}, A_{\kappa}^{y}\right]_{n m},
\end{aligned}
$$

where $Q_{\kappa}=\sum_{l}^{\mathrm{empty}}\left|u_{\kappa l}\right\rangle\left\langle u_{\kappa l}\right|$. In the second line the completeness relation was used to obtain an expression containing the Berry connection and Berry curvature matrices $A_{\kappa n m}^{x}$ and $\Omega_{\kappa n m}^{x y}$ for the occupied states, defined in terms of the slab orbitals by equations like Eq. (16) but with the replacements $l n \rightarrow n$ and $l^{\prime} m \rightarrow m$. The matrix in Eq. (35) is known as the non-Abelian Berry curvature [27], and it follows from its gauge covariance that Eq. (33a) can be written in any HWF gauge as

$$
\theta_{2}=-\int d \kappa \operatorname{Tr}\left(Z_{\kappa} \widetilde{\Omega}_{\kappa}^{x y}\right),
$$

where Eq. (33b) for $\widetilde{\Omega}_{\kappa}^{x y}$ has been replaced with Eq. (35). Returning to the maximally localized HWF gauge,

$$
\theta_{2}=-\int d \kappa \sum_{n} z_{\kappa n} \widetilde{\Omega}_{\kappa n n}^{x y} .
$$

In Sec. II E 2 we will recast this expression in a form similar to Eq. (18) for $\theta_{3}$, but for the moment (and also for numerics; see Sec. III A) Eq. (37) is more convenient.

Equations (36) and (37) constitute the central result of this section. We emphasize that nowhere in their derivations was it assumed that the Chern numbers $C_{n}$ of the individual Wannier sheets must vanish.

Let us conclude with a discussion of the conditions needed in order for $\theta_{2}$ to be a well-defined quantity. Under the transformation (27), Eq. (37) changes by

$$
\Delta \theta_{2}=-2 \pi C_{\text {slab }} \Delta z,
$$

where $C_{\text {slab }}=\sum_{n} C_{n}$ is the Chern number of the entire slab, and we used the fact that the trace of the commutator in Eq. (35) vanishes. Contrary to $\Delta \theta_{0}$ which is guaranteed to vanish for any crystallite, $\Delta \theta_{2}$ is nonzero for quantum anomalous Hall slabs where $C_{\text {slab }}$ is a nonzero integer. Clearly, $\theta_{2}$ is ill-defined in such systems.

How can one reconcile $\Delta \theta_{2} \neq 0$ for a quantum anomalous Hall slab with $\Delta \theta_{0}=0$ for a crystallite cut from the same slab? To understand this result, we invoke the definition

$$
C(\boldsymbol{r})=-4 \pi \operatorname{Im}\langle\boldsymbol{r}|P x Q y| \boldsymbol{r}\rangle
$$

of the "local Chern marker" [28] to rewrite Eq. (28) as

$$
\Delta \theta_{0}=-2 \pi\left[\int d \boldsymbol{r} C(\boldsymbol{r})\right] \Delta z,
$$

which can be directly compared with Eq. (38) for $\Delta \theta_{2}$. Whenever $C_{\text {slab }} \neq 0, \int d \boldsymbol{r} C(\boldsymbol{r})$ vanishes in a nontrivial manner, with extensive contributions from the edges of the crystallite exactly canceling those from the interior [28]. When we switched from open to periodic boundary conditions along $\hat{\boldsymbol{x}}$ and $\hat{\boldsymbol{y}}$ to obtain Eq. (32), the edges were suppressed. Clearly, that step relied on the absence of extensive edge contributions to $\int d \boldsymbol{r} C(\boldsymbol{r})$, or equivalently,

$$
C_{\text {slab }}=0 .
$$

\section{E. Surface theorem for the CSA coupling}

\section{Statement of the theorem}

Armed with Eq. (37) for the CSA coupling of an insulating slab of thickness $L_{z}=N_{z} c$, let us relate it to the bulk coupling $\theta_{3}$ by considering the thermodynamic limit. Defining the dimensionless slab CSA coupling

$$
\theta_{\text {slab }} \equiv \frac{\theta_{2}}{N_{z} c}
$$

and reasoning by analogy with Eq. (29), one might expect that

$$
\theta_{\text {slab }} \stackrel{?}{\rightarrow} \theta_{3} \text { for } N_{z} \rightarrow \infty .
$$

This cannot be quite correct, because $\theta_{3}$ is only defined modulo $2 \pi$ whereas $\theta_{\text {slab }}$ is uniquely defined. Turning this observation around, it should be possible to resolve the quantum of ambiguity in $\theta_{3}$ by isolating quantized surfacelike contributions to $\theta_{\text {slab }}$.

It is useful to divide the slab conceptually into three regions (Fig. 1): a bottom surface region I, an interior region II containing precisely $N_{\text {II }}$ bulk unit cells, and a top surface region III. The boundaries are chosen to respect the HWF sheets, so that each sheet belongs uniquely to one region. We assume that the surface regions occupy nonextensive fractions of the volume of the slab, but are nonetheless sufficiently thick to ensure that the cuts $z_{0}$ and $z_{0}+N_{\mathrm{II}} c$ fall in bulklike regions.

We will prove the following assertion, valid when the Fermi level lies in a gap common to both the bulk and surface bands:

$$
\theta_{\text {slab }} \rightarrow \theta_{3}-2 \pi C_{\mathrm{III}} \text { for } N_{z} \rightarrow \infty \text {, }
$$

with $\theta_{3}$ expressed in terms of the bulklike HWFs in region II by Eq. (18), and $C_{\mathrm{III}}=\sum_{n \in \mathrm{III}} C_{n}$ the total integer Chern number of the HWFs ascribed to the top surface region. The Chern indices of the valence-band manifold are assumed to vanish in all three lattice directions, which implies [see Eq. (19)]

$$
C_{\mathrm{II}}=\sum_{n \in \mathrm{II}} C_{n}=N_{\mathrm{II}} \sum_{n} C_{0 n}=0 .
$$

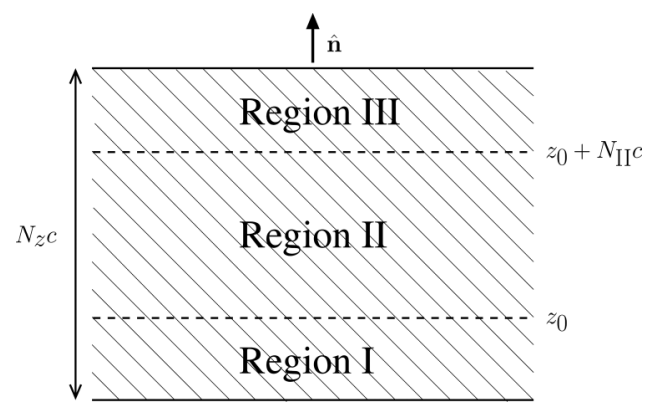

FIG. 1. Sketch of the slab configuration discussed in the text (adapted from Ref. [19]). The HWFs are localized in the surfacenormal direction $\hat{\boldsymbol{n}}=\hat{z}$. 
From Eq. (41) we also require $C_{\mathrm{I}}+C_{\mathrm{II}}+C_{\mathrm{III}}=0$, so that

$$
C_{\mathrm{I}}+C_{\mathrm{III}}=0 \text {. }
$$

Once the assignment of the slab HWF sheets to the three regions has been decided, the two terms on the right-hand side of Eq. (44) become uniquely defined. That is, Eq. (18) must be evaluated using the choice of unit cell consistent with the $N_{\text {II }}$ bulk cells in region II, and the leftover sheets then contribute to $C_{\mathrm{I}}$ and $C_{\mathrm{III}}$. If a different assignment is made, an integer multiple of $2 \pi$ may get transferred between the two contributions to Eq. (44). We will assume that a specific assignment has been made that satisfies two rules:

(1) Sheets belonging to a given region have sequential indices $n$, with $z_{\kappa n} \leqslant z_{\kappa, n+1}$.

(2) The last sheet belonging to region I does not touch the first sheet belonging to region II.

Because region II comprises an integer number of bulk cells, the second rule also implies that the topmost sheet in region II does not touch the first sheet in region III, guaranteeing that the Chern numbers of the two surface regions are well defined. This should be possible to arrange in most cases-even at critical parameter values where two Wannier sheets touch at a high-symmetry point-by a judicious choice of the first sheet in region II. (One exception is a $\mathbb{Z}_{2}$-odd topological insulator protected by $\mathcal{T}$ symmetry, where there are no gaps between bulk Wannier sheets; see, for example, the third panel in Fig. 1 of Ref. [20]. Before applying the current analysis to such a system, one would have to break $\mathcal{T}$ symmetry slightly to gap the spectrum.)

Equation (44) can be understood as follows. The assignment of the HWFs to the three regions decides which ones belong to a given unit cell in the interior region. This removes the only gauge freedom that is capable of affecting the branch choice for the phase angle $\theta_{3}$ in Eq. (18). The extra term $-2 \pi C_{\text {III }}$ gathers the contributions to the slab CSA coupling from any leftover sheets after tiling the bulk cell towards the top surface. This is analogous to the electric polarization of a slab [19], where the relevant quantized quantity carried by the Wannier sheets is the charge rather than the Chern number. (One difference is that Wannier sheets can have different Chern numbers, while every sheet carries the same charge $-e$.)

\section{Proof of the theorem}

In order to establish Eq. (44), we start from the left-hand side. Using Eqs. (37) and (35) and exchanging indices $n$ and $m$ in one term we find, approximating the slab width $N_{z} c$ by the width $N_{\mathrm{II}} c$ of the interior region,

$$
\begin{aligned}
\theta_{\text {slab }} & =-\frac{1}{c} \int d \boldsymbol{\kappa} \vartheta_{\text {slab }}, \\
\vartheta_{\text {slab }} & =\frac{1}{N_{\text {II }}} \sum_{n}\left[z_{n} \Omega_{n n}^{x y}+i \sum_{m}\left(z_{m}-z_{n}\right) A_{n m}^{x} A_{m n}^{y}\right],
\end{aligned}
$$

where the label $\boldsymbol{\kappa}$ has been dropped from the integrand. (Here and for the rest of this section, it is understood that certain equalities only hold exactly for $N_{\mathrm{II}} \rightarrow \infty$.) The similarity to Eq. (18) for $\theta_{3}$ is apparent.
Next we decompose $\theta_{\text {slab }}$ and $\vartheta_{\text {slab }}$ into contributions from each region,

$$
\theta_{\text {slab }}=\theta_{\mathrm{I}}+\theta_{\mathrm{II}}+\theta_{\mathrm{III}},
$$

by restricting the summation over $n$ in Eq. (47b) to the sheets assigned to that region (the index $m$ is still allowed to run over all Wannier sheets in the slab).

Let us start with the top surface region. The contribution to $\vartheta_{\text {III }}$ from the second term in Eq. (47b) vanishes for a thick slab, because (i) that term involves the relative coordinate $z_{m}-$ $z_{n}$, (ii) $A_{n m}^{x} A_{m n}^{y}$ drops exponentially to zero when $\left|z_{m}-z_{n}\right|^{2}$ is much larger than the HWF spread $\left\langle z_{n}\right\rangle^{2}-\left\langle z_{n}\right\rangle^{2}$, and (iii) region $A$ is nonextensive. We are left with the first term in Eq. (47b), which involves the absolute coordinate $z_{n}$. In the limit $N_{\mathrm{II}} \rightarrow \infty$ we can set $z_{n} \rightarrow z_{0}+N_{\mathrm{II}} c$ for $n \in$ III (see Fig. 1) to obtain

$$
\theta_{\mathrm{III}}=-2 \pi\left(1+\frac{z_{0}}{N_{\mathrm{II}} c}\right) C_{\mathrm{IIII}} .
$$

Similarly, $\theta_{\mathrm{I}}=-2 \pi\left(z_{0} / N_{\mathrm{II}} c\right) C_{\mathrm{I}}$. Adding the two together and using Eq. (46) yields the net surface contribution

$$
\theta_{\text {surf }}=\theta_{\mathrm{I}}+\theta_{\mathrm{III}}=-2 \pi C_{\mathrm{III}},
$$

which is origin-independent and quantized in units of $2 \pi$.

Now we turn to the contribution from the interior region. The quantity $\vartheta_{\text {II }}$ can be approximated as

$$
\vartheta_{\mathrm{II}}=\frac{1}{N_{\mathrm{II}}}\left[\sum_{n \in \mathrm{II}} z_{n} \Omega_{n n}^{x y}+i \sum_{n m \in \mathrm{II}}\left(z_{m}-z_{n}\right) A_{n m}^{x} A_{m n}^{y}\right] .
$$

By also restricting the summation over $m$ to region II, we have dropped nonextensive contributions. Since that region is bulklike, we can switch to the notation of Sec. II B. Replacing $n \rightarrow l n$ and $m \rightarrow l^{\prime} m$ and invoking Eqs. (15) and (16), the first term in Eq. (51) becomes

$$
\vartheta_{z \Omega}=\sum_{n} z_{0 n} \Omega_{0 n, 0 n}^{x y}
$$

[we have dropped a term $\left(1 / N_{\mathrm{II}}\right) \sum_{l}(l c) \sum_{n} \Omega_{0 n, 0 n}^{x y}$ that vanishes upon integration over $\kappa$ by virtue of Eq. (19)]. As for the second term, it becomes

$$
\vartheta_{\Delta x y}=i \sum_{l n m}\left(z_{l m}-z_{0 n}\right) A_{0 n, l m}^{x} A_{l m, 0 n}^{y} .
$$

Adding the two and integrating over $\boldsymbol{\kappa}$ we find, comparing with Eq. (18),

$$
\theta_{\mathrm{II}}=\theta_{z \Omega}+\theta_{\Delta x y}=\theta_{3} .
$$

Combining Eqs. (48), (50), and (54) we arrive at Eq. (44), completing the proof of the surface theorem. Note that condition (19) insures that the two terms on the right-hand side of Eq. (44) are separately origin independent.

\section{LAYERED HALDANE MODEL}

In this section we illustrate the preceding discussion with a concrete example: a 3D tight-binding model of an insulator that pumps a quantum of CSA coupling during a cyclic adiabatic evolution. 
The model consists of a stack of half-filled Haldane-model [29] ("haldanium") layers placed exactly on top of each other, that are allowed to interact via interlayer hoppings. The on-site energies are equipped with a dependence on a cyclic tuning parameter $\phi$, such that if the layers were decoupled, their Chern numbers would either be all zero or alternate between +1 and -1 from one layer to the next, depending on the value of $\phi$. (An isolated layer with nonzero Chern number $C$ in the valence band is a quantum anomalous Hall insulator with $\mathrm{AHC}$ $\sigma_{y x}=C e^{2} / h$.)

For decoupled layers, the system would pass through metallic points at critical $\phi$ values where the Chern numbers change. By introducing $\phi$-dependent interlayer couplings, it is possible to keep the bulk insulating throughout the cycle. The band touchings at the critical $\phi$ values are then replaced with touchings between Wannier sheets residing mostly on individual layers, accompanied by a transfer of Chern number (the total Chern number of the valence bands vanishes in all three directions). Each Wannier sheet participates in two touching events per cycle, one with the sheet below and another with the sheet above, resulting in the net transfer across a unit cell of a Chern number of -1 .

The numerical results presented below were obtained using the PythTB code package [30].

\section{A. Cyclic evolution of a bilayer model}

We begin by considering a model consisting of only two coupled haldanium layers. Although there is no CSA pumping in this model, it serves to illustrates the elementary sheettouching events during a cyclic evolution, and provides the building blocks for the full 3D model.

The Hamiltonian of a single layer $p$ is

$$
\begin{aligned}
H_{p}^{(0)}= & (-1)^{p} \Delta \sum_{i} \tau_{i} c_{p i}^{\dagger} c_{p i}+t_{1} \sum_{\langle i j\rangle} c_{p i}^{\dagger} c_{p j} \\
& +(-1)^{p} t_{2} \sum_{\langle i j\rangle}\left(i c_{p i}^{\dagger} c_{p j}+\text { H.c. }\right),
\end{aligned}
$$

where indices $i$ and $j$ label sites on the $A$ and $B$ sublattices marked in Fig. 2(a), and $\tau_{i}=+1(-1)$ for $i \in A(B)$. The first and second terms contain the on-site energies and the nearestneighbor hoppings, respectively, and the third describes a pattern of staggered magnetic fluxes generated by complex
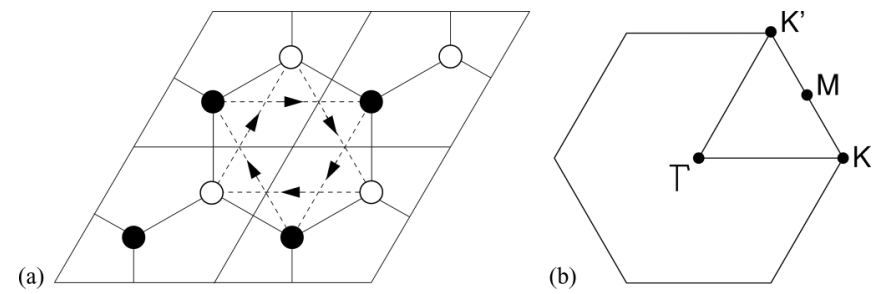

FIG. 2. (a) Four unit cells of an isolated haldanium layer. Sites on the $A(B)$ sublattices are marked with open (filled) circles, and the arrows indicate the directions of the second-nearest-neighbor hoppings in Eq. (55) with amplitudes $i(-1)^{p} t_{2}$. (b) First Brillouin zone, with high-symmetry points marked. (Reproduced from Ref. [31].) When viewing (b) as the projected BZ of a layered 3D model in Sec. III B, the labels of the high-symmetry points become $\bar{\Gamma}, \overline{\mathrm{M}}, \overline{\mathrm{K}}$, and $\overline{\mathrm{K}}^{\prime}$. second-neighbor hoppings; $\langle\langle i j\rangle\rangle$ denotes pairs of sites for which the hopping from $j$ to $i$ has amplitude $i(-1)^{p} t_{2}$, and "H.c." stands for "Hermitian conjugate". In the following we choose $t_{2}>0$, and use it to set the energy scale for the model.

The Hamiltonian of the coupled bilayer system is

$$
H_{\text {bilayer }}=\sum_{p=1,2} H_{p}^{(0)}+t_{3} \sum_{i} \tau_{i}\left(c_{1 i}^{\dagger} c_{2 i}+\text { H.c. }\right),
$$

where $p=1$ and $p=2$ denote the layers at $z=0$ and $z=c / 2$, respectively, and sites on different layers with the same index $i$ are aligned vertically. Thus, the first term describes two copies of the Haldane model with opposite signs for both the on-site energies and the complex hoppings, and the second couples them via vertical hoppings that alternate in sign between the two sublattices.

We parametrize the model as

$$
\begin{aligned}
t_{1} & =-4 t_{2}, \\
t_{3} & =t_{2}, \\
\Delta & =(3 \sqrt{3}+2 \cos \phi) t_{2} .
\end{aligned}
$$

Referring to the phase diagram of the Haldane model [29], the layer Chern numbers in the decoupled limit $t_{3} \rightarrow 0$ are

$$
C_{p}= \begin{cases}(-1)^{p-1} & \text { for } \phi \in[\pi / 2,3 \pi / 2] \bmod 2 \pi, \\ 0 & \text { otherwise. }\end{cases}
$$

When $\phi=\pi / 2 \bmod \pi$, the energy gap closes at $\mathrm{K}$ on both layers. The addition of interlayer hoppings reopens the gap, ensuring that at half filling the system remains insulating for all values of $\phi$, with zero Chern number for the group of two valence bands.

Although Chern numbers can no longer be defined for the individual layers when $t_{3} \neq 0$, they are "inherited" by the Wannier sheets. The energy gap, Wannier centers at $K$, and Wannier-sheet Chern numbers are shown in the top and middle panels of Fig. 3 over one cycle, $\phi \in[0,2 \pi]$. Initially, the Chern numbers vanish for both sheets. The sheets themselves are very flat, and sit close to one of the layers at $z=0$ or $z=c / 2$. With increasing $\phi$ they approach one another, at $\phi=\pi / 2$ they touch at $\mathrm{K}$ (but not at $\mathrm{K}^{\prime}$ ) changing their Chern numbers to \pm 1 , and then separate again. There is no closure of the energy gap during this process. In the second half of the cycle the system retraces the same parameter-space path in the opposite direction.

The CSA coupling has been calculated in two different ways: for the periodic $2 \mathrm{D}$ crystal using Eq. (37) for $\theta_{2}$ (discretizing the covariant Berry curvature on a $120 \times 120 k$ point mesh, following Ref. [25]), and for finite flakes using Eq. (20) for $\theta_{0}$. The results are compared via Eq. (29), further dividing both sides by $c$ (twice the interlayer separation) in order to obtain a dimensionless coupling. In practice we evaluate $\theta_{2}(N)=\theta_{0} / N^{2} A_{\text {cell }}$, where $\theta_{0}$ is for a flake containing $N \times N$ primitive cells, and then extrapolate to $N \rightarrow \infty$ the results obtained for $N=10,15,20,25,30$, by fitting to the expression [25]

$$
\theta_{2}(N)=\theta_{2}(\infty)+a / N+b / N^{2} .
$$

The evolution of $\theta_{2} / c$ with $\phi$ is shown in the bottom panel of Fig. 3. It starts very small but nonzero, and reaches a maximum 


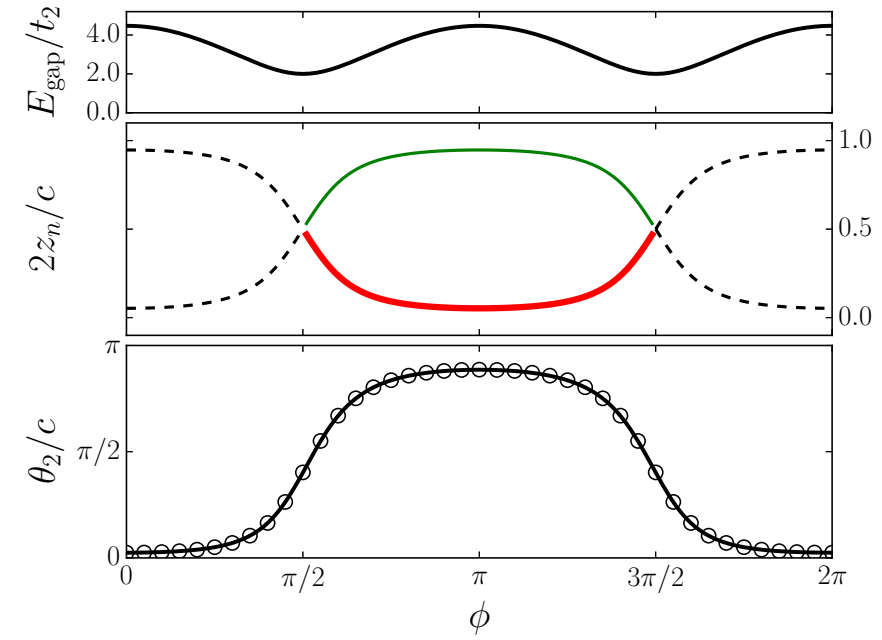

FIG. 3. Numerical results for the bilayer model as a function of the adiabatic loop parameter $\phi$. Top: Minimum energy gap between the second (highest occupied) and third bands; the minimum gap is always at point $\mathrm{K}$ in the $2 \mathrm{D} \mathrm{BZ}$. Middle: Wannier charge centers at $\mathrm{K}$, in units of the layer separation $c / 2$. A dashed line indicates a Wannier sheet with Chern number $C=0$, a heavy (red) solid line denotes $C=+1$, and a light (green) solid line denotes $C=-1$. The lower (upper) sheet has index $n=1(n=2)$. Bottom: Dimensionless CSA coupling $\theta_{2} / c$. Open circles, extrapolation of $\theta_{2}(N) / c$ from finite-size samples. Solid line, direct calculation using the $k$-space formula (37).

of close to $\pi$ halfway through the cycle. The precise agreement between the two curves confirms the correctness of the $k$ space formula (37). Importantly, the agreement persists in the range $\pi / 2<\phi<3 \pi / 2$ where the Wannier sheets have Chern numbers \pm 1 .

\section{B. Cyclic evolution of the bulk model}

The bulk model with two layers per cell and vertical lattice constant $c$ is constructed by repeating the bilayer model along $z$ :

$$
H_{\text {bulk }}=\sum_{p}\left[H_{p}^{(0)}+t_{3, p} \sum_{i} \tau_{i}\left(c_{p i}^{\dagger} c_{p+1, i}+\text { H.c. }\right)\right],
$$

where the layer index $p$ now runs over all integers. The intralayer hoppings and on-site energies are still given by Eqs. (57a) and (57c). If we were to use Eq. (57b) for the interlayer hoppings, at half filling the valence and conduction bands would touch at point $\mathrm{H}$ in the $3 \mathrm{D} \mathrm{BZ}$ for $\phi=\pi / 2 \mathrm{mod}$ $\pi$. In order to keep the gap open at all $\phi$, we modulate the interlayer hoppings out of phase with the on-site energies,

$$
t_{3, p}=\left[1+(-1)^{p-1} \gamma \sin \phi\right] t_{2}
$$

with $\gamma=0.4$. Each layer is more strongly coupled to the layer below during half of the cycle, and to the layer above during the other half. The insulating loop forms an ellipse on the $\left(\Delta, t_{3, p}\right)$ plane, with a metallic point at the center.

To demonstrate the pumping behavior of this model we have calculated $\theta_{3}(\phi)$ from Eq. (18). The HWFs and the charge centers were obtained from a parallel-transport construction applied to strings of $k$ points along $k_{z}$ [21].

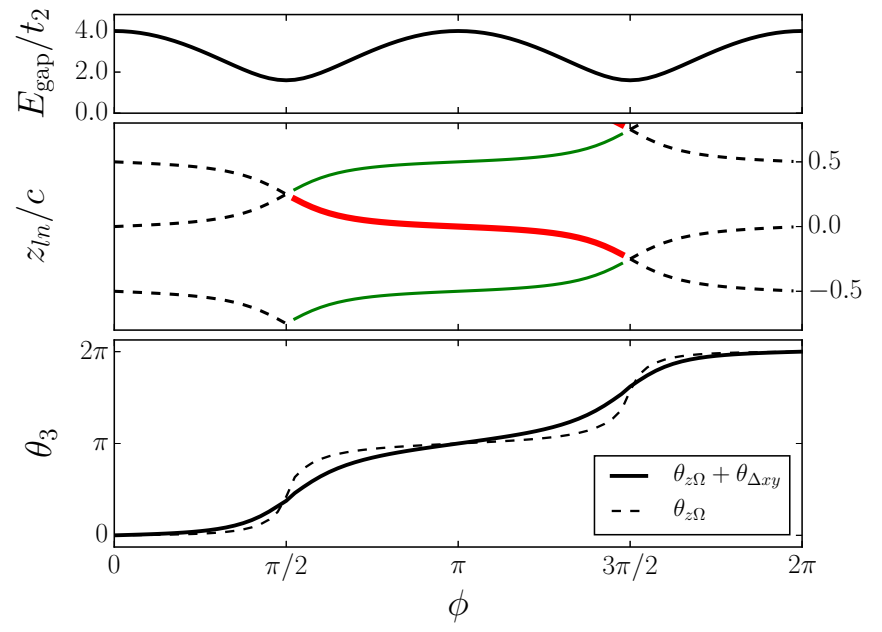

FIG. 4. Numerical results for the bulk model as a function of the adiabatic loop parameter $\phi$. Top: Minimum energy gap between the second and third bands; the minimum gap is always at point $\mathrm{H}$, which projects onto $\overline{\mathrm{K}}$ in the surface BZ. Middle: Periodically repeated Wannier charge centers at $\overline{\mathrm{K}}$, in units of the lattice constant $c$; the dashed line and colored heavy/light lines have the same meaning as in Fig. 3. The lower Wannier sheet has indices $l=-1$ and $n=2$, the middle one $l=0$ and $n=1$, and the upper one $l=0$ and $n=$ 2. Bottom: Total CSA coupling $\theta_{3}=\theta_{z \Omega}+\theta_{\Delta x y}$, and the term $\theta_{z \Omega}$ responsible for pumping.

The results obtained with a mesh of $120 \times 120 \times 6$ points in the BZ are shown in Fig. 4. The energy gap remains open throughout the cycle. In the first half, the evolution of the two Wannier centers in the home cell $l=0$ resembles that in Fig. 3 for the bilayer system. At point $\overline{\mathrm{K}}$ in the projected BZ they start off at 0 and $c / 2$, respectively, begin approaching until at $\phi=\pi / 2$ they touch at $z=c / 4$ exchanging one unit of Chern number, and then drift apart, recovering the initial separation at $\phi=\pi$. By then $\theta_{3}$ has increased continuously from 0 to $\pi$, with the term $\theta_{\Delta x y}$ going through zero at $\phi=0, \pi / 2$, and $\pi$.

Thanks to the modulation in the interlayer hoppings, the second half of the cycle is very different from that of the bilayer. Instead of reconnecting with their original partners, the Wannier centers continue to drift in the same direction until at $\phi=3 \pi / 2$ they touch the periodic images of their original partners in the adjacent cells. As a result $\theta_{3}$ continues to increase, reaching $2 \pi$ by the end of the cycle.

At $\phi=0 \bmod \pi$ the model acquires extra symmetry [32]: each layer becomes a mirror plane, forcing $\theta_{3}$ to be an integer multiple of $\pi[13,24]$. According to Fig. $4, \theta_{3}=0$ at $\phi=0$ and $\theta_{3}=\pi$ at $\phi=\pi$. In the CSA pump model of Ref. [20], the same values occurred at the beginning and in the middle of the pumping cycle, where they were protected by $\mathcal{T}$ rather than mirror symmetry. In Appendix A we analyze further the quantization of $\theta_{3}$ due to mirror symmetry in our model, and show that at $\phi=\pi$ it becomes a topological crystalline insulator with a single surface Dirac cone on any surface normal to the mirror plane.

The fact that the CSA coupling is pumped by $2 \pi$ over an adiabatic cycle signals that the occupied band manifold of the 4D Bloch Hamiltonian $H_{\text {bulk }}\left(k_{x}, k_{y}, k_{z}, \phi\right)$ has a nonvanishing second Chern number [10]. In Appendix B we map the second 
Chern number of the model over an augmented parameter space.

\section{Cyclic evolution of the slab interior, keeping the surfaces gapped}

Let us now study the cyclic evolution in a slab geometry. If the entire slab-including the surfaces-returns to the initial state at the end of the cycle, it must pass through a state with metallic surfaces [20]. This scenario will be investigated in the next section, but first we examine here what happens when a surface modification is introduced to avoid the gap closure at the surfaces. To this end we adjust the on-site energies on the top and bottom layers of the slab according to

$$
\Delta=(3 \sqrt{3}-2) t_{2} \text { for } \pi<\phi \leqslant 2 \pi,
$$

so that in the second half of the cycle the on-site energies on the two surface layers remain frozen at the values reached at $\phi=\pi$, while on all other layers they continue to evolve according to Eq. (57c). Thus, at $\phi=2 \pi$ the surfaces are in a different insulating state than at $\phi=0$. According to the surface theorem the CSA couplings of the two configurations may differ by a multiple of $2 \pi$, and this is indeed what happens for our model.

We consider a slab containing $N_{z}$ repetitions in the $z$ direction of the model defined by Eq. (60), and use Eq. (37) to calculate $\theta_{\text {slab }}(\phi)$ [Eq. (42)] at half filling. Figure 5 shows the results obtained with a ten-layer slab, i.e., $N_{z}=5$. Thanks to the surface modification, the energy gap shown in the

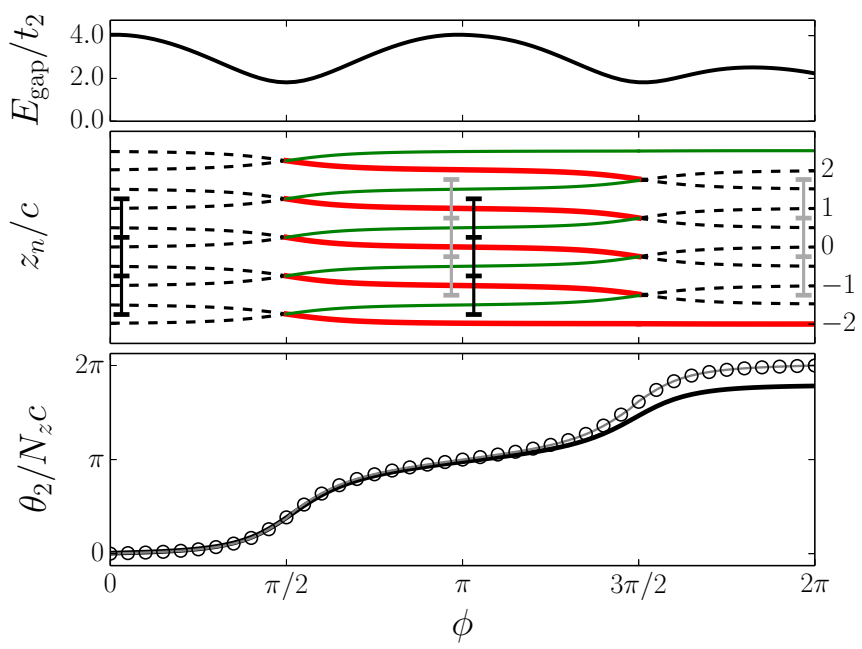

FIG. 5. Numerical results for a ten-layer slab $\left(N_{z}=5\right)$ as a function of the adiabatic loop parameter $\phi$. The surface Hamiltonian has been modified according to Eq. (62), to keep the surface insulating throughout the cycle. Top: Minimum energy gap (always at point $\mathrm{K}$ in the 2D BZ) at half filling. Middle: Wannier charge centers at $\mathrm{K}$ in units of the lattice constant $c$, with indices ranging from $n=1$ (bottom) to $n=10$ (top); the dashed line and colored heavy/light lines have the same meaning as in Fig. 3. Two possible choices of bulk cells are indicated by the gray and black lines. Bottom: Dimensionless CSA coupling $\theta_{\text {slab }}=\theta_{2} / N_{z} c$. Heavy line, calculation done at $N_{z}=5$. Open circles, extrapolation to $N_{z} \rightarrow \infty$ of calculations done at $N_{z}=4,7,10$. Light gray line, bulk CSA coupling $\theta_{3}$ taken from Fig. 4. top panel remains open throughout the cycle. As seen in the middle panel, all ten Wannier sheets start out with zero Chern number. The eight subsurface Wannier sheets show a bulklike behavior, switching partners between $\phi=\pi / 2$ and $\phi=3 \pi / 2$ as in Fig. 4. This leaves the top and bottom sheets unpaired and with Chern numbers -1 and +1 , respectively, until the end of the cycle. The slab CSA coupling (heavy line in the lower panel) goes from zero at $\phi=0$ to somewhat less than $2 \pi$ at $\phi=2 \pi$. It is only when $N_{z} \rightarrow \infty$ (open circles) that $\theta_{\text {slab }}(2 \pi) \rightarrow 2 \pi$; this is similar to an adiabatic charge pump, where the exact quantization of particle transport only occurs in the thermodynamic limit [33].

The result $\theta_{\text {slab }}(2 \pi)-\theta_{\text {slab }}(0)=2 \pi$ in the limit of a thick slab can be readily understood from the surface theorem. Since $H_{\text {bulk }}(\phi)$ is periodic, the sheet structure in the interior of the slab is identical at $\phi=0$ and at $\phi=2 \pi$, so that $\theta_{3}(0)=\theta_{3}(2 \pi)$ for any choice of bulk cell (two possible choices are indicated in Fig. 5; since all the bulk sheets have zero Chern number, the value of $\theta_{3}$ does not depend on that choice [20]). What changes between the two states of the slab is the excess Chern number that remains after tiling the chosen cell towards the top surface: inspection of Fig. 5 shows that $C_{\mathrm{III}}(0)=0$, while $C_{\mathrm{III}}(2 \pi)=-1$. Referring to Eq. (44),

$$
\left.\Delta \theta_{\text {slab }}\right|_{\phi=0} ^{\phi=2 \pi}=-\left.2 \pi \Delta C_{A}\right|_{\phi=0} ^{\phi=2 \pi}=2 \pi .
$$

Let us now use Eq. (44) to determine $\theta_{\text {slab }}$ in the range $\pi / 2<$ $\phi<3 \pi / 2$ where the Wannier sheets have alternating Chern numbers \pm 1 . When switching from the gray to the black cells in Fig. 5, the sheet with $C=+1$ (heavy red line) in the central cell stays the same, while the one with $C=-1$ (light green line) gets replaced by its periodic image below, changing $\theta_{3}$ by $-2 \pi$. Tiling the former cell towards the upper surface leaves no excess sheets, so that $C_{\mathrm{III}}=0$ and $\theta_{\text {slab }}=\theta_{3}$. With the latter choice there is one leftover sheet with $C_{\mathrm{III}}=-1$ that contributes $+2 \pi$ to $\theta_{\text {slab }}$, exactly canceling the change in $\theta_{3}$. The calculated value of $\theta_{\text {slab }}$ is therefore the same with both cell choices.

\section{Cyclic evolution of the entire slab}

Next we consider a truly cyclic evolution of the slab, where not only the interior but also the surfaces return to their initial state. Because $\theta_{\text {slab }}(\phi)$ is uniquely determined by $H_{\text {slab }}(\phi)$ and the band filling, it must return at $\phi=2 \pi$ to the same value it had at $\phi=0$. The way this happens for our model is shown in Fig. 6. From $\phi=0$ to $\phi_{c}=3 \pi / 2$ the CSA coupling of a thick slab (open circles) evolves exactly as in Fig. 5. Then at the critical parameter $\phi_{c}$ it drops abruptly by $2 \pi$, switching between two branches of the bulk CSA coupling $\theta_{3}$ (light gray lines).

At $\phi_{c}$, the energy gap closes at the two surfaces. In order to avoid spurious interactions between the surfaces that reopen the gap slightly and complicate the analysis, let us switch to a semi-infinite geometry with a single surface. This is obtained by iteratively constructing the retarded Green's function in a local basis [34] and then tracing out the surface states. Figure 7 shows the spectral function in the vicinity of point $\overline{\mathrm{K}}$ in the surface BZ, evaluated by tracing over sites in the topmost two layers. The gap closing at $\phi_{c}$ (top panel) consists of a linear crossing between two surface bands. Moving slightly 


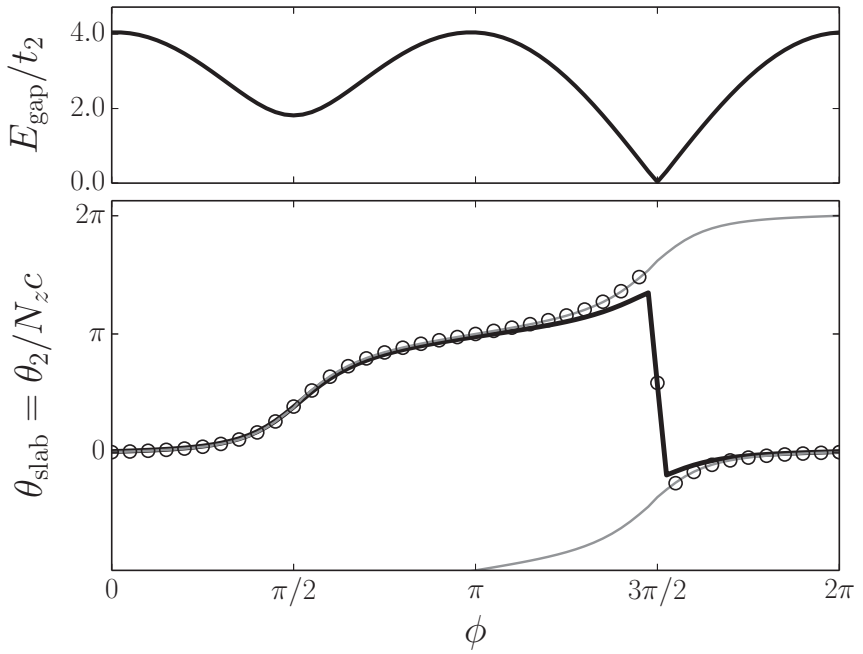

FIG. 6. Same as the top and bottom panels of Fig. 5, except that now the entire slab, including the surfaces, undergoes a cyclic evolution.

away from $\phi_{c}$ (bottom panel), the crossing becomes avoided. Just like the touching events between Wannier sheets seen in Figs. 3-5, this band touching constitutes a Weyl point in the space of parameters $\left(k_{x}, k_{y}, \phi\right)$. Since the states that cross are localized at the surface, we call it a "surface Weyl point".

The discontinuous $2 \pi$ drop in $\theta_{\text {slab }}$ occurs because at $\phi_{c}$ $\mathrm{a}-2 \pi$ quantum of Berry flux is channeled from the valence to the conduction bands through the surface Weyl point. This removes the exact amount of Berry curvature that gets pushed to the surface during one pumping cycle, allowing the surface AHC to return to its initial value by the end of the cycle. Referring to the middle panel of Fig. 5 (but without the bottom surface, due to the semi-infinite geometry), the effect of the surface Weyl point would be to add at $\phi_{c}=3 \pi / 2$ a Chern

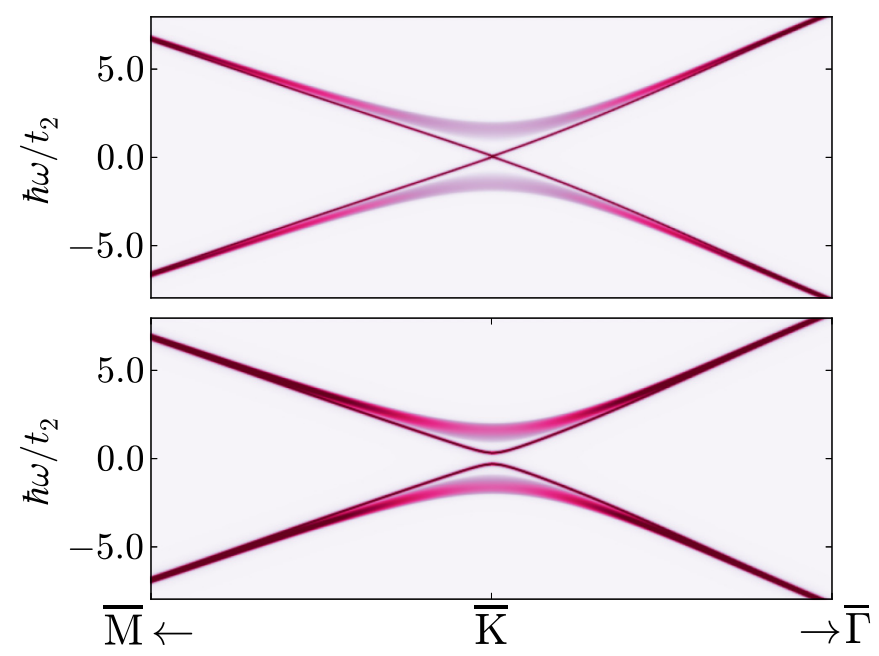

FIG. 7. Surface spectral function in a semi-infinite geometry, calculated at the critical parameter value $\phi_{c}=3 \pi / 2$ where the CSA coupling in Fig. 6 changes discontinuously (top panel), and at $\phi=\phi_{c} \pm \pi / 20$ (bottom panel). The spectral function is plotted along two lines that extend $(1 / 20)$ th of the distance from $\overline{\mathrm{K}}$ to $\overline{\mathrm{M}}$ and $\bar{\Gamma}$. amount of +1 to the topmost Wannier sheet, changing its Chern number from -1 to zero without it touching the sheet below.

The scenario sketched here, in which a single Weyl point in $\left(k_{x}, k_{y}, \phi\right)$ space disposes of the excess Chern number pumped to the surface during a cycle that also returns the surface Hamiltonian to itself, is not the only possibility. In general there could be a finite interval in $\phi$ over which the surface becomes metallic. In such cases, however, we would still expect that the "Fermi surface in $\left(k_{x}, k_{y}, \phi\right)$ space" should always enclose such a Weyl point [35], or more precisely, a set of Weyl points with a net chirality equal to the second Chern number characterizing the pumping cycle.

\section{SUMMARY}

In summary, we have examined how the presence of insulating surfaces fixes the quantized part of the CSA coupling that is undefined for a purely bulk band insulator. In the basis of HWFs maximally localized along the surface-normal direction, the CSA coupling of a thick slab becomes a sum of two terms: (i) a nonquantized contribution (previously found in Ref. [20]) associated with the bulklike HWFs far from the surfaces, and (ii) a quantized contribution given by the excess Chern number of the Wannier sheets near the surface. When some of the bulk Wannier sheets have nonzero Chern numbers the individual terms in this decomposition become dependent on the choice of bulk cell, but their sum remains unique (for a given surface termination).

Inspired by the representation of the CSA pumping process in the HWF basis, where $2 \pi$ quanta of Berry curvature are passed from sheet to sheet [20], we constructed a 3D tightbinding model for a quantum CSA pump by coupling quantum anomalous Hall layers with tunable Chern numbers. In order to illustrate the surface theorem of Eq. (44), the CSA coupling strength of the model, as well as the charge centers and Chern numbers of the individual Wannier sheets, were tracked during cyclic evolutions carried out in different geometries (periodic bulk crystal, finite slab, and semi-infinite crystal). These numerical studies revealed how the Berry curvature pumped across the bulk is extracted at the surfaces, when the surface Hamiltonian also undergoes a cyclic evolution.

At two isolated points along the cycle, our model acquires mirror symmetry. The two mirror-symmetric states are topologically distinct: it is not possible to go from one to the other along a path in parameter space that preserves mirror symmetry without closing and reopening the direct band gap. The trivial state has $\theta_{3}=0 \bmod 2 \pi$ (corresponding to a quantized surface AHC of $\left.n e^{2} / h\right)$, while the nontrivial one has $\theta_{3}=\pi \bmod 2 \pi$ and a half-quantized surface AHC, and these are the only values consistent with mirror symmetry. This is analogous to the quantization of the bulk polarization and "surface" (edge) bound charge to integer and half-integer multiples of $e$, respectively, at the two inversion-symmetric points along a charge-pumping cycle in 1D [19].

The HWF representation has become a powerful tool for identifying topological phases in 2D and 3D [22,36-39]. While in previous studies the objects of interest were the bulk HWFs, the present work shows that, as in the $1 \mathrm{D}$ polarization problem, 
in $3 \mathrm{D}$ additional topological information can be extracted from the HWFs near the surface.

\section{ACKNOWLEDGMENTS}

The Center for Nanostructured Graphene (CNG) is sponsored by the Danish National Research Foundation, Project No. DNRF103. D.V. acknowledges support from NSF Grant No. DMR-1408838. I.S. acknowledges support from European Commission Grant No. CIG-303602, and from Grant No. FIS2016-77188-P from the Spanish Ministerio de Economía y Competitividad.

\section{APPENDIX A: QUANTIZATION OF THE CSA COUPLING IN THE PRESENCE OF MIRROR SYMMETRY}

In this appendix we use the HWF picture to analyze how the presence of mirror symmetry $M_{z}$ in our bulk model at $\phi=0$ and $\phi=\pi$ forces the CSA coupling to be either 0 or $\pi$ modulo $2 \pi$, as seen in Fig. 4. Henceforth we use the symbol $\theta_{3}^{M}$ to denote the value of the CSA coupling when mirror symmetry is present.

Mirror symmetry forces the term $\theta_{\Delta x y}$ in Eq. (18c) to vanish, because that term is odd under $M_{z}$ and single valued. Therefore, $\theta_{3}^{M}$ is fully determined by Eq. (18b),

$$
\theta_{3}^{M}=-\frac{1}{c} \int d \kappa \sum_{n} z_{\kappa 0 n} \Omega_{\kappa, 0 n, 0 n}^{x y} .
$$

According to Fig. 4, at $\phi=0$ and $\phi=\pi$ the two Wannier centers at $\overline{\mathrm{K}}$ in the home cell of our model coincide with one of the two mirror planes, $z=0$ or $z=1 / 2$ (in units of the lattice constant $c$ ). In fact, the two Wannier sheets are pinned to the mirror planes for all values of $\boldsymbol{\kappa}$. Taking the Wannier centers out of the integrand in the previous equation and using Eq. (17), we find

$$
\theta_{3}^{M}=-\pi C_{1 / 2},
$$

where $C_{1 / 2}$ is the Chern number of the Wannier sheet pinned at $z=1 / 2$. Consulting again the middle panel of Fig. 4 we see that $C_{1 / 2}=0$ at $\phi=0$ and $C_{1 / 2}=-1$ at $\phi=\pi$, so that $\theta_{3}^{M}=0$ and $\theta_{3}^{M}=\pi$, respectively, in agreement with the lower panel of the same figure.

Alternatively, the band topology protected by mirror symmetry can be characterized by "mirror Chern numbers" $C_{k_{z}}^{M}$ defined on the mirror-invariant planes in the BZ [40] (for this model, they are $k_{z}=0$ and $k_{z}=\pi$, in units of $1 / c$ ). The two descriptions are related by [24]

$$
\theta_{3}^{M}=\pi\left(\left|C_{0}^{M}\right|+\left|C_{\pi}^{M}\right|\right) \bmod 2 \pi .
$$

Below we verify this relation explicitly for our model. We make use of the fact that $\left|C_{k_{z}}^{M}\right|$ counts the number of Dirac cones along the projection line of the $k_{z}$ plane onto the surface BZ of a mirror-symmetric surface [40].

Figure 8 shows the surface spectral function calculated at $\phi=0, \pi$ using a semi-infinite geometry that respects mirror symmetry (surface normal to the $y$ direction, corresponding to zigzag edges for the individual layers). The surface BZ is a rectangle on the $\left(k_{x}, k_{z}\right)$ plane, with $\bar{\Gamma}=(0,0)$ at the center, $\overline{\mathrm{R}}=\left(\frac{1}{2}, \frac{1}{2}\right)$ at the corner, and $\overline{\mathrm{X}}=\left(\frac{1}{2}, 0\right)$ and $\overline{\mathrm{Z}}=\left(0, \frac{1}{2}\right)$ at the centers of the two edges.

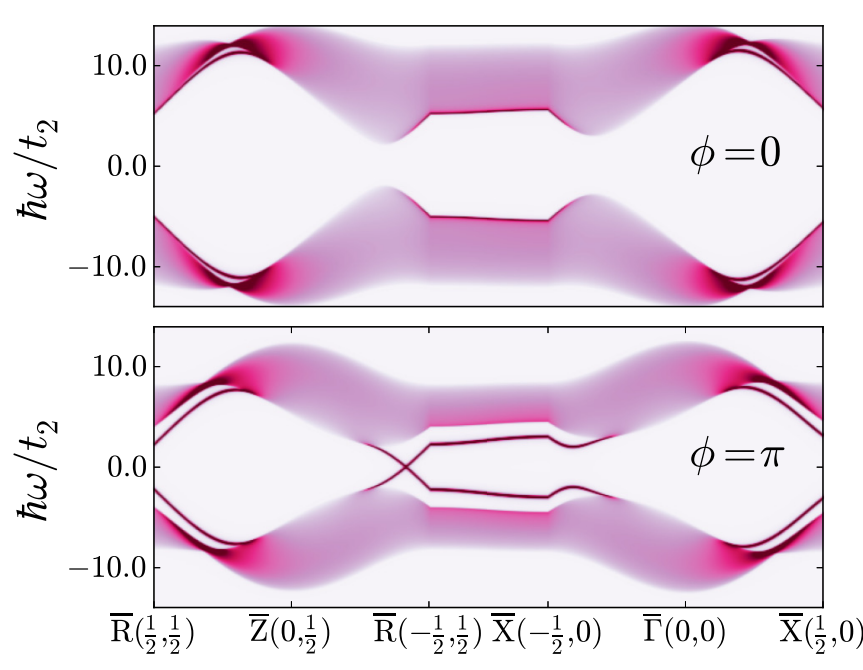

FIG. 8. Surface spectral function of the layered model at $\phi=0$ and $\phi=\pi$, for a semi-infinite geometry with the surface orthogonal to the $y$ direction. In both cases the entire system (bulk plus surface) has mirror symmetry, but while in the top panel the bulk CSA coupling vanishes, in the bottom panel it equals $\pi$ and protects a surface Dirac cone.

At $\phi=0$ (top panel of Fig. 8), the absence of surface states traversing the bulk gap implies $\left|C_{0}^{M}\right|=\left|C_{\pi}^{M}\right|=0$, in agreement with the value $\theta_{3}=0$. At $\phi=\pi$ (bottom panel) there are two such surface bands of opposite chirality. They cross along the projection $\overline{\mathrm{Z}} \overline{\mathrm{R}}$ of the $k_{z}=\pi$ plane so that $\left|C_{\pi}^{M}\right|=1$ and $\left|C_{0}^{M}\right|=0$, consistent with $\theta_{3}=\pi$. In conclusion, the model at $\phi=0$ is a trivial insulator while at $\phi=\pi$ it is a topological crystalline insulator with a single Dirac cone on any surface normal to the mirror plane.

\section{APPENDIX B: MAPPING THE SECOND CHERN NUMBER IN AN AUGMENTED PARAMETER SPACE}

In the same way that a quantum charge pump is characterized by a nonzero (first) Chern number defined over a two-dimensional parameter space $(k, \lambda)$ [33], a quantum CSA pump has a nonvanishing second Chern number in the four-dimensional space of $\boldsymbol{k}$ and the pumping parameter [10], i.e., $\left(k_{x}, k_{y}, k_{z}, \phi\right)$ for the model of Eq. (60).

In order to vary the behavior of the loop so that it can also represent trivial cycles, we introduce an additional parameter $\beta$ in the interlayer hoppings of the model,

$$
t_{3, p}=\left[1+(-1)^{p-1} \gamma(\sin \phi+2 \sin \beta)\right] t_{2} .
$$

The case of $\beta=0$ corresponds to Eq. (61). The 4D Bloch Hamiltonian $H_{\text {bulk }}\left(k_{x}, k_{y}, k_{z}, \phi\right)$ has four Dirac points at which the top two valence bands and bottom two conduction bands all become degenerate at the $\mathrm{H}$ point in the 3D BZ for $\phi=$ $3 \pi / 2$. These occur at $\beta= \pm \pi / 6$ and $\beta= \pm 5 \pi / 6$, delineating different regions of $\beta$ with different second Chern numbers. In particular, the second Chern number is \pm 1 when $\beta \in$ $[-\pi / 6, \pi / 6]$ or $\beta \in[5 \pi / 6,7 \pi / 6]$, respectively, and is zero otherwise. This is illustrated in Fig. 9, where the three cases of $\beta \in\{0, \pi / 6, \pi / 2\}$ are shown. The change from a trivial to a nontrivial pumping cycle at $\beta=\pi / 6$ is depicted in the two bottom panels. In the second half of the cycle the bulk HWFs 

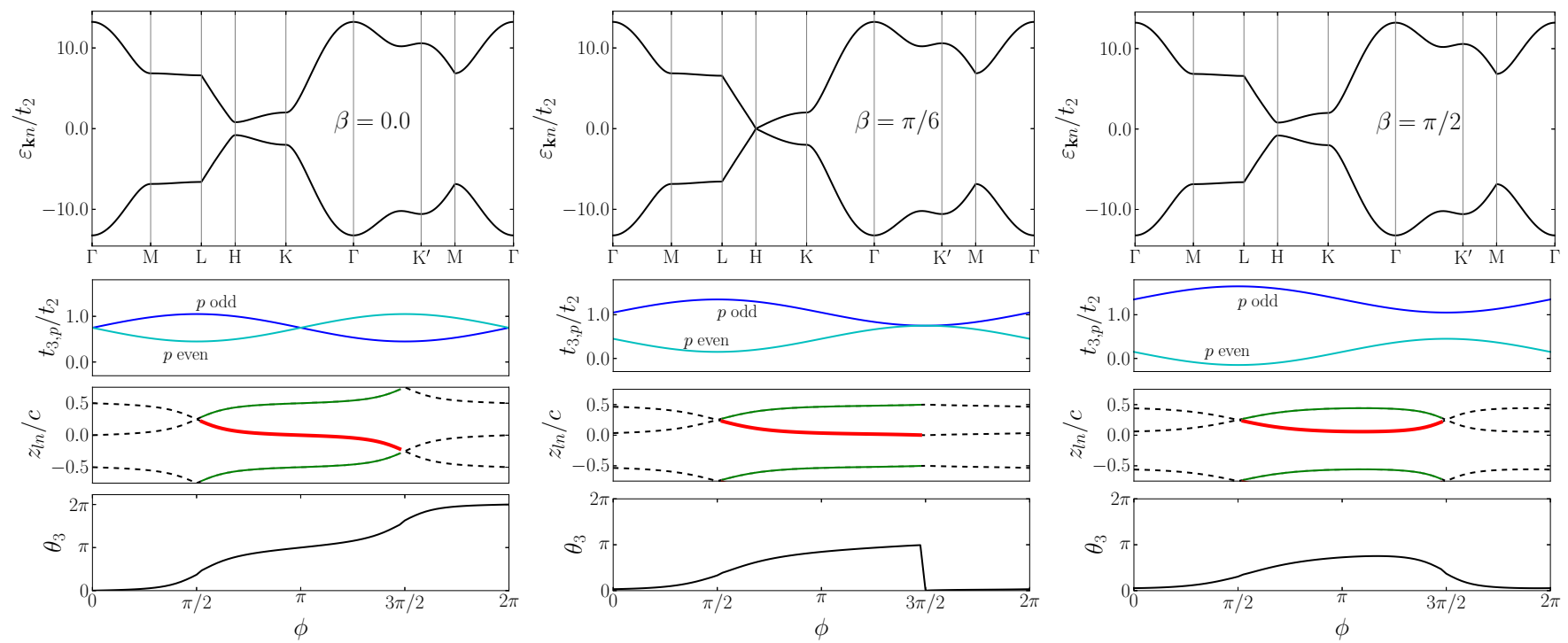

FIG. 9. Top: Band structures in the 3D BZ, calculated at $\phi=3 \pi / 2$ with three different values of $\beta$. The bands are everywhere twofold degenerate, and at $\beta=\pi / 6$ they become fourfold degenerate at $\mathrm{H}$, forming a Dirac point. Bottom: Interlayer couplings, hybrid Wannier charge centers at point $\overline{\mathrm{K}}$ in the projected BZ, and bulk CSA coupling, plotted as a function of $\phi$ for the same three values of $\beta$. In the panels depicting the Wannier centers, the dashed lines and colored heavy/light lines have the same meaning as in Fig. 3.

remain localized near the atomic layers, and at $\phi=3 \pi / 2$ they exchange Berry flux with the two conduction bands (rather than with one another) through the Dirac point, changing their Chern numbers from $\{+1,-1\}$ to $\{0,0\}$. In this process, the net Berry flux through the nonchiral Dirac point vanishes as expected.

Alternatively, one can view the same process in terms of a 5D Hamiltonian $H_{\text {bulk }}\left(k_{x}, k_{y}, k_{z}, \phi, \beta\right)$ where the second Chern number is calculated on $4 \mathrm{D}$ hyperslices corresponding to fixed values of $\beta$. When $\beta$ passes through the four Dirac points, the second Chern number of the slices changes by one. The Dirac points in 5D are the analogs of Weyl points in 3D; indeed, the integer obtained by integrating the second Chern class on a closed 4D hypersurface surrounding the degeneracy point is nothing other than the change in the second Chern number of the hyperslices.
[1] R. D. Peccei and H. R. Quinn, CP Conservation in the Presence of Pseudoparticles, Phys. Rev. Lett. 38, 1440 (1977).

[2] J. M. Pendlebury, S. Afach, N. J. Ayres, C. A. Baker, G. Ban, G. Bison, K. Bodek, M. Burghoff, P. Geltenbort, K. Green, W. C. Griffith, M. van der Grinten, Z. D. Grujic, P. G. Harris, V. Helaine, P. Iaydjiev, S. N. Ivanov, M. Kasprzak, Y. Kermaidic, K. Kirch, H. C. Koch, S. Komposch, A. Kozela, J. Krempel, B. Lauss, T. Lefort, Y. Lemiere, D. J. R. May, M. Musgrave, O. Naviliat-Cuncic, F. M. Piegsa, G. Pignol, P. N. Prashanth, G. Quemener, M. Rawlik, D. Rebreyend, J. D. Richardson, D. Ries, S. Roccia, D. Rozpedzik, A. Schnabel, P. Schmidt-Wellenburg, N. Severijns, D. Shiers, J. A. Thorne, A. Weis, O. J. Winston, E. Wursten, J. Zejma, and G. Zsigmond, Revised experimental upper limit on the electric dipole moment of the neutron, Phys. Rev. D 92, 092003 (2015).

[3] S. Weinberg, A New Light Boson? Phys. Rev. Lett. 40, 223 (1978).

[4] F. Wilczek, Problem of Strong $P$ and $T$ Invariance in the Presence of Instantons, Phys. Rev. Lett. 40, 279 (1978).

[5] S. J. Asztalos, G. Carosi, C. Hagmann, D. Kinion, K. van Bibber, M. Hotz, L. J. Rosenberg, G. Rybka, J. Hoskins, J. Hwang, P. Sikivie, D. B. Tanner, R. Bradley, and J. Clarke, SQUID-Based Microwave Cavity Search for Dark-Matter Axions, Phys. Rev. Lett. 104, 041301 (2010).
[6] F. Wilczek, Two Applications of Axion Electrodynamics, Phys. Rev. Lett. 58, 1799 (1987).

[7] E. Witten, Fermion path integrals and topological phases, Rev. Mod. Phys. 88, 035001 (2016).

[8] G. Gabadadze and M. Shifman, QCD vaccum and axions: What's happening? Int. J. Mod. Phys. A 17, 3689 (2002).

[9] F. Wilczek, Particle physics and condensed matter: The saga continues, Phys. Scr. T168, 014003 (2016).

[10] X.-L. Qi, T. L. Hughes, and S.-C. Zhang, Topological field theory of time-reversal invariant insulators, Phys. Rev. B 78, 195424 (2008).

[11] X. Wan, A. M. Turner, A. Vishwanath, and S. Y. Savrasov, Topological semimetal and Fermi-arc surface states in the electronic structure of pyrochlore iridates, Phys. Rev. B 83, 205101 (2011).

[12] L. Balents, Viewpoint: Weyl electrons kiss, Physics 4, 36 (2011).

[13] C. Fang, M. J. Gilbert, and B. A. Bernevig, Bulk topological invariants in noninteracting point group symmetric insulators, Phys. Rev. B 86, 115112 (2012).

[14] S. Coh, D. Vanderbilt, A. Malashevich, and I. Souza, ChernSimons orbital magnetoelectric coupling in generic insulators, Phys. Rev. B 83, 085108 (2011).

[15] A. Malashevich, I. Souza, S. Coh, and D. Vanderbilt, Theory of orbital magnetoelectric response, New J. Phys. 12, 053032 (2010). 
[16] A. M. Essin, A. M. Turner, J. E. Moore, and D. Vanderbilt, Orbital magnetoelectric coupling in band insulators, Phys. Rev. B 81, 205104 (2010).

[17] A. M. Essin, J. E. Moore, and D. Vanderbilt, Magnetoelectric Polarizability and Axion Electrodynamics in Crystalline Insulators, Phys. Rev. Lett. 102, 146805 (2009).

[18] R. D. King-Smith and D. Vanderbilt, Theory of polarization of crystalline solids, Phys. Rev. B 47, 1651 (1993).

[19] D. Vanderbilt and R. D. King-Smith, Electric polarization as a bulk quantity and its relation to surface charge, Phys. Rev. B 48, 4442 (1993).

[20] M. Taherinejad and D. Vanderbilt, Adiabatic Pumping of ChernSimons Axion Coupling, Phys. Rev. Lett. 114, 096401 (2015).

[21] N. Marzari and D. Vanderbilt, Maximally localized generalized Wannier functions for composite energy bands, Phys. Rev. B 56, 12847 (1997).

[22] M. Taherinejad, K. F. Garrity, and D. Vanderbilt, Wannier center sheets in topological insulators, Phys. Rev. B 89, 115102 (2014).

[23] The expression for $\theta_{\Delta x y}$ in Eq. (12) of Ref. [20] has a typo in it: A minus sign is missing.

[24] D. Varjas, F. de Juan, and Y.-M. Lu, Bulk invariants and topological response in insulators and superconductors with nonsymmorphic symmetries, Phys. Rev. B 92, 195116 (2015).

[25] D. Ceresoli, T. Thonhauser, D. Vanderbilt, and R. Resta, Orbital magnetization in crystalline solids: Multiband insulators, Chern insulators, and metals, Phys. Rev. B 74, 024408 (2006).

[26] E. I. Blount, Formalisms of band theory, Solid State Phys. 13, 305 (1962).

[27] C. A. Mead, The geometric phase in molecular systems, Rev. Mod. Phys. 64, 51 (1992).

[28] R. Bianco and R. Resta, Mapping topological order in coordinate space, Phys. Rev. B 84, 241106 (2011).

[29] F. D. M. Haldane, Model for a Quantum Hall Effect without Landau Levels: Condensed-Matter Realization of the "Parity Anomaly", Phys. Rev. Lett. 61, 2015 (1988).
[30] Available at http://physics.rutgers.edu/pythtb/.

[31] T. Thonhauser and D. Vanderbilt, Insulator/Chern-insulator transition in the Haldane model, Phys. Rev. B 74, 235111 (2006).

[32] In addition to mirror symmetry $M_{z}$, the bulk model at $\phi=0, \pi$ also exhibits $C_{2}^{y} * \mathcal{T}$ symmetry, with the twofold axis lying on the plane halfway between two atomic layers and pointing along a zigzag edge. This additional symmetry, which also constrains $\theta_{3}$ to be an integer multiple of $\pi$, can be removed by changing the nearest-neighbor hopping amplitude along one of the two in-plane directions. We have checked that doing so modifies the $\theta_{3}(\phi)$ curve, but does not affect the quantized values at $\phi=0, \pi$.

[33] D. J. Thouless, Quantization of particle transport, Phys. Rev. B 27, 6083 (1983).

[34] M. P. L. Sancho, J. M. L. Sancho, and J. Rubio, Highly convergent schemes for the calculation of bulk and surface Green functions, J. Phys. F 15, 851 (1985).

[35] D. Gosálbez-Martínez, I. Souza, and D. Vanderbilt, Chiral degeneracies and Fermi-surface Chern numbers in bcc Fe, Phys. Rev. B 92, 085138 (2015).

[36] L. Fu and C. L. Kane, Time reversal polarization and a $Z_{2}$ adiabatic spin pump, Phys. Rev. B 74, 195312 (2006).

[37] A. A. Soluyanov and D. Vanderbilt, Computing topological invariants without inversion symmetry, Phys. Rev. B 83, 235401 (2011).

[38] R. Yu, X. L. Qi, A. Bernevig, Z. Fang, and X. Dai, Equivalent expression of $\mathbb{Z}_{2}$ topological invariant for band insulators using the non-Abelian Berry connection, Phys. Rev. B 84, 075119 (2011).

[39] D. Gresch, G. Autès, O. V. Yazyev, M. Troyer, D. Vanderbilt, B. A. Bernevig, and A. A. Soluyanov, Z2Pack: Numerical implementation of hybrid Wannier centers for identifying topological materials, arXiv:1610.08983

[40] J. C. Y. Teo, Liang Fu, and C. L. Kane, Surface states and topological invariants in three-dimensional topological insulators: Application to $\mathrm{Bi}_{1-x} \mathrm{Sb}_{x}$, Phys. Rev. B 78, 045426 (2008). 